\title{
Herbal medicine for the management of polycystic ovary syndrome (PCOS) and associated oligo/ amenorrhoea and hyperandrogenism; a review of the laboratory evidence for effects with corroborative clinical findings
}

\author{
Susan Arentz ${ }^{1 *}$, Jason Anthony Abbott ${ }^{2}$, Caroline Anne Smith ${ }^{3}$ and Alan Bensoussan ${ }^{3}$
}

\begin{abstract}
Background: Polycystic ovary syndrome (PCOS) is a prevalent, complex endocrine disorder characterised by polycystic ovaries, chronic anovulation and hyperandrogenism leading to symptoms of irregular menstrual cycles, hirsutism, acne and infertility. Evidence based medical management emphasises a multidisciplinary approach for PCOS, as conventional pharmaceutical treatment addresses single symptoms, may be contra-indicated, is often associated with side effects and not effective in some cases. In addition women with PCOS have expressed a strong desire for alternative treatments. This review examines the reproductive endocrine effects in PCOS for an alternative treatment, herbal medicine. The aim of this review was to identify consistent evidence from both pre-clinical and clinical research, to add to the evidence base for herbal medicine in PCOS (and associated oligo/amenorrhoea and hyperandrogenism) and to inform herbal selection in the provision clinical care for these common conditions.

Methods: We undertook two searches of the scientific literature. The first search sought pre-clinical studies which explained the reproductive endocrine effects of whole herbal extracts in oligo/amenorrhoea, hyperandrogenism and PCOS. Herbal medicines from the first search informed key words for the second search. The second search sought clinical studies, which corroborated laboratory findings. Subjects included women with PCOS, menstrual irregularities and hyperandrogenism.

Results: A total of 33 studies were included in this review. Eighteen pre-clinical studies reported mechanisms of effect and fifteen clinical studies corroborated pre-clinical findings, including eight randomised controlled trials, and 762 women with menstrual irregularities, hyperandrogenism and/or PCOS. Interventions included herbal extracts of Vitex agnus-castus, Cimicifuga racemosa, Tribulus terrestris, Glycyrrhiza spp., Paeonia lactiflora and Cinnamomum cassia. Endocrine outcomes included reduced luteinising hormone $(\mathrm{LH})$, prolactin, fasting insulin and testosterone. There was evidence for the regulation of ovulation, improved metabolic hormone profile and improved fertility outcomes in PCOS. There was evidence for an equivalent effect of two herbal medicines and the pharmaceutical agents bromocriptine (and Vitex agnus-castus) and clomiphene citrate (and Cimicifuga racemosa). There was less robust evidence for the complementary combination of spirinolactone and Glycyrrhiza spp. for hyperandrogenism.

Conclusions: Preclinical and clinical studies provide evidence that six herbal medicines may have beneficial effects for women with oligo/amenorrhea, hyperandrogenism and PCOS. However the quantity of pre-clinical data was limited, and the quality of clinical evidence was variable. Further pre-clinical studies are needed to explain the effects of herbal medicines not included in this review with current clinical evidence but an absence of pre-clinical data.
\end{abstract}

\footnotetext{
*Correspondence: s.arentz@uws.edu.au

${ }^{1}$ National Institute of Complementary Medicine, University of Western,

Locked Bag 1797, Penrith South, NSW 2751 Sydney, Australia

Full list of author information is available at the end of the article
} 


\section{Background}

Polycystic ovary syndrome (PCOS) is a complex, common reproductive and endocrine disorder affecting up to $17.8 \%$ of reproductive aged women [1]. Medical management places strong emphasis on a multidisciplinary approach as pharmaceutical treatments appear to be only moderately effective in treating individual symptoms [2,3]. Conventional pharmaceutical management is limited by the prevalence of contraindications in women with PCOS [3], non-effectiveness in some circumstances [4], side effects [5] and by preferences of women with PCOS for alternatives to pharmaceutical management [6]. This review examines the mechanisms of effect for a potential alternative treatment, herbal medicine, and reveals six herbal medicines with both pre-clinical and clinical data explaining the reproductive endocrinological effects in PCOS and associated oligo/amenorrhoea and hyperandrogenism.

Complementary medicine (CM) use by women has increased during the past ten years [7-11] with rates of use ranging between $26 \%$ and $91 \%[8,9]$. One of the popular types of CM is herbal medicine [11,12]. Herbal medicines are known to contain pharmacologically active constituents with physiological effects on female endocrinology and have been positively associated with reduced incidences of breast cancer, osteoporosis and cardiovascular disease [13-18].

PCOS is a life-long condition and although the exact cause is yet to be identified, it is believed to have epigenetic origins, influenced by the uterine environment and behavioural factors [19]. Being overweight exacerbates all aspects of PCOS due to underlying metabolic disturbances [3]. Signs and symptoms are mediated by hormonal disorder including elevated androgens and fasting insulin, and abnormal relative ratio of the gonadotropins luteinising hormone (LH) and follicle stimulating hormone (FSH) [19]. Endocrine imbalances occur within the framework of disordered ovarian folliculogenesis, chronic anovulation, clinical signs of hyperandrogenism and metabolic syndrome [19].

Pharmaceutical treatment for menstrual irregularity includes the oral contraceptive pill (OCP) and ovulation induction with clomiphene citrate (clomiphene) [20,21] depending on fertility needs. Women with PCOS are however likely to exhibit contraindications for the OCP [3] and whilst induction of ovulation with clomiphene has demonstrated success, pregnancy rates remain inexplicably low [4]. Up to thirty $30 \%$ of women, particularly overweight women with PCOS, fail to respond to clomiphene therapy $[4,22,23]$. Management for hyperandrogenism includes anti-androgens and hypoglycaemic pharmaceuticals such as metformin [24]. Metfomin has demonstrated effectiveness for improving insulin sensitivity and hyperandrogenism, however use of metformin is associated with the high incidence of adverse effects including nausea, vomiting and gastro-intestinal disturbances [5].

Herbal medicines are complex interventions with the potential for synergistic and antagonistic interactions between compounds [25]. Effects within the body may also exhibit complexity by simultaneous interactions with various body systems, both biochemically and by altering organ function [26]. The focus of this review was studies investigating whole herbal medicine extracts with direct effects on reproductive endocrinology for the treatment of women with irregular menstruation, hyperandrogenism and PCOS. The rationale for using this methodology was to identify herbal medicines with current scientific evidence explaining specific reproductive endocrinological effects in PCOS, oligo/amenorrhoea and hyperandrogenism, to develop understanding for the direct effects of herbal medicines on reproductive endocrinology and to highlight herbal medicines for which there was current scientific evidence supporting herbal medicine selection. The purpose of this review is to inform clinical decisions in integrative settings and meet clinicians and consumers preferences for pragmatic herbal management within an holistic, individualised treatment frame [27,28].

We compared laboratory evidence including scientific studies using cell culture and animal models, with clinical data for proof-of-concept effects. A narrative synthesis of pre-clinical studies explaining reproductive endocrinological effects for herbal medicines with corroborative clinical evidence is presented.

\section{Methods}

We used the following definitions. PCOS according to the Rotterdam diagnostic criteria, specified by the presence of two out of three features; oligo/amenorrhoea, hyperandrogenism and polycystic ovaries on ultrasound [29,30]. Associated endocrine features for PCOS included elevated $\mathrm{LH}$ [31], which is strongly associated with infertility ( $\mathrm{p}=0.0003)$ [32] and miscarriage [33] and elevated fasting glucose which is prevalent in approximately $31 \%$ of women with PCOS including normal weight women [34].

Oligomenorrhoea was defined as menstrual cycle length that extended beyond 35 days (day one being the first day of menses). Amenorrhoea was defined as no menstrual period for three to six months or more [19]. This review was focussed on hypothalamic, pituitary and ovarian causes of menstrual irregularity with associated elevated gonadotropins including $\mathrm{LH}$ and prolactin and arrested folliculogenesis typically observed in polycystic ovaries. Hyperprolactinaemia is usually considered a unique cause for oligo/amenorrhoea; however in the present case it was included due to the potential co-existence for elevated prolactin, LH and PCOS, [32,35].

Hyperandrogenism was defined as clinical or biochemically excessive androgens. Clinical markers in females 
include cutaneous manifestations such as the presence of acne, hirsutism and/or male pattern alopecia. Biochemical indications include elevated plasma concentration of androgens.

We conducted two searches. The first was sensitive and aimed to capture all pre-clinical studies explaining the reproductive endocrine effects of whole herbal extracts in PCOS or associated oligo/amenorrhoea and hyperandrogenism. The second search was specific and sought only clinical studies investigating herbal medicines revealed during the first search (for which a mechanism of effect had been demonstrated). We additionally searched, on a case by case basis for pre-clinical evidence for herbal medicines identified during the second search, but not included in the results of the first search. Clinical studies were excluded based on the absence of evidence for a mechanism of effect for the whole herbal extract in reproductive endocrinology associated with PCOS, oligo/amenorrhoea and hyperandrogenism. We used this approach to improve transparency and to limit confirmation bias for herbal medicines favoured by the authors in clinical practice.

The first search revealed ten herbal medicines with a demonstrated mechanism of reproductive endocrinological effect for the whole herbal extract in PCOS, oligo/amenorrhoea and hyperandrogenism. These were Cimicifuga racemosa, Cinnamomum cassia, Curcuma longa, Glycyrrhiza spp., Matricaria chamomilla, Mentha piperita, Paeonia lactiflora, Silybum marianum, Tribulus terrestris and Vitex agnus-castus. Herbal medicines with a demonstrated mechanism of effect were entered as key terms in the second search.

We searched the following electronic databases: the Cochrane Library, MEDLINE ovidSP, CINAHL (1936 to present), SciVerse, EMBASE, PubMed, from the date of database inception to June 2014. In addition, we manually searched bibliographies of review articles.

Key terms for the first search included: title or abstract CONTAINS 'herbal medicine' OR 'herbal extract"' OR 'phytotherapy' OR 'botanical' AND title or abstract CONTAINS 'androgen*' OR 'oestrogen*'OR 'follicle stimulating hormone' OR 'luteinising hormone' OR 'prolactin' OR 'insulin' OR 'glucose' OR 'polycystic 'ovar"'. Search terms for the second search included the following key words in the title or abstract, CONTAINS; 'menstrual irregularity' OR 'oligomenorrhoea' OR 'amenorrhoea' OR 'hyperandrogenism' OR 'hirsutism' OR 'acne', OR ‘polycystic ovary syndrome' OR 'PCOS' OR 'polycystic ovar"' OR 'oligoovulation' OR 'anovulation' OR 'fertility' OR 'infertility' OR 'pregnancy' AND ten herbal medicines identified from the laboratory search; 'Cimicifuga racemosa' OR 'Cinnamomum cassia' OR 'Curcuma longa' OR 'Glycyrrhiza 'OR Matricaria chamomilla OR 'Mentha piperita' OR 'Paeonia lactiflora' OR 'Silybum marianum' OR 'Tribulus terrestris'
OR 'Vitex agnus-castus'. Truncation was used to capture plural key words and synonyms, and acronyms were used for some hormones (FSH and $\mathrm{LH}$ ).

Our laboratory search included investigations into the effects of herbal medicine using computer models, cell cultures, animals with PCOS induced with oestradiol valerate and androgens and sterilised and ovariectomised rats. We excluded laboratory studies which commenced using isolated chemicals not directly extracted from crude herbal medicines and studies examining androgen effects in male animals.

Our second search for clinical trials was performed without language restriction and included randomised controlled trials, non-randomised, open label and single arm clinical trials. We included clinical studies investigating commercially available herbal extracts and investigations that compared the effectiveness of herbal medicine with pharmaceuticals. We excluded clinical studies investigating herbal medicines with unrelated outcomes (including premenstrual syndrome, endometriosis and mastalgia) and clinical studies examining the effectiveness of complex herbal formulas for PCOS and associated oligo/amenorrhoea and hyperandrogenism, without demonstration of a mechanism of effect for the whole complex formula. We compared data from laboratory and animal studies with the outcomes of clinical trials. Clinical studies were assessed for risks of bias at study and outcome levels with risks summarised, tabulated (Tables 1 and 2) and presented in contextual narrative.

\section{Results}

\section{Laboratory studies}

Our search identified 33 laboratory (pre-clinical) studies (Figure 1). Eighteen studies met the inclusion criteria, nine reported on receptor binding assays or ovarian or pituitary (brain) cell cultures, [36-44] and nine used an animal experimental model with hormone assays and/or post-mortem examination of ovarian, uterine and brain histology, [45-53] (Table 1). We excluded 15 studies for the following reasons; investigation of effects in male animals $(n=4)$ and investigations which commenced with constituents that were isolated from herbal medicines $(n=5)$. Six studies were excluded due to no clinical evidence found $(n=6)$.

\section{Clinical studies}

Following the electronic and manual searches of bibliographies, forty six clinical studies were identified for inclusion/exclusion assessment (Figure 1). A pre-requisite for the inclusion of clinical studies was identified laboratory evidence explaining the mechanism of effect in reproductive endocrinology. Fifteen met the inclusion criteria [54-68]. Eight were randomised controlled trials (RCTs) including 762 women [61-68] (Table 2). Thirty one studies were excluded for the following reasons; investigation of 
Table 1 Summary of evidence for the reproductive endocrinological effects of six herbal medicines in oligo/amenorrhoea, hyperandrogenism and PCOS

\begin{tabular}{lll}
\hline Herbal medicine $\quad$ Evidence & Physiological effects in menstrual \\
\cline { 2 - 4 } & irregularity (oligo/amenorrhoea),
\end{tabular}

\begin{tabular}{llll} 
Botanic name & Pre-clinical in vitro and in vivo & Clinical RCTs (detailed in Table 2) irregularity (oligo/amenorrhoea), \\
\cline { 2 - 4 } & hyperandrogenism and/or PCOS.
\end{tabular}

\section{Herbal extract}

Vitex agnus-castus

Eight studies investigated gonadotropic hormone

Ethanol extracts

concentration effects of Vitex agnus-castus.

Strontan ${ }^{\oplus}$, Mastodynon ${ }^{\oplus}$,

Phyto-hypophyson ${ }^{\oplus}$

Agnacaston ${ }^{\oplus}$

Cimicifuga racemosa

Ethanol extract

Klimadynon ${ }^{\odot}$
1. Investigation for equivalence of dopaminergic effects for Vitex agnus-castus and the pharmaceutical Lisuride using rat pituitary cell cultures (basal and stimulated cells) [41]

2. Brain (calf, guinea pig and rats) receptor tissue cultures including DA2, histamine and $5 \mathrm{HT}$ transporters. Radio ligand and super-fusion experiments [40]

3. Three investigations found affinity for Vitex agnus-castus and $\beta$ oestrogen receptors $[38,43,69]$

4. Using recombinant human dopamine (DA2) recepto proteins [38]

5. The affinity of Vitex agnus-castus extract (with and without fatty acids) for human $\mu$ opoid receptor cells cloned and transfected into hamster ovary cells [70]

6. The endocrine effects for Vitex agnus-castus were investigated in normal and ovariectomised rats [49]

7. Corpus striatum membrane including D2 receptors to assess the inhibitory properties of Vitex agnus-castus on prolactin, FSH and LH [39]

Four laboratory studies investigated pituitary oestrogen receptor binding and gonadotropin concentrations following exposure to Cimicifuga racemosa.

1. One study investigated a constituent flavonoid of Cimicifuga racemosa, discovered during the course of the study for reduction for $\mathrm{LH}$ in ovariectomised rats [37]

2. Oestrogen receptor binding affinity for Cimicifuga racemosa was studied using pituitary cell cultures from ovariectomised rats. This study followed a clinical study demonstrating significantly lowered LH in post-

menopausal women following administration of

Cimicifuga racemosa (2 mg for two months) against placebo control $(n=110)[45]$

3. Binding affinity for oestrogen receptors (ERa) for Cimicifuga racemosa examined using MCF7 cell cultures [44]

4. Chronic and acute dosage effects of Cimicifuga racemosa and oestradiol on oestrogen receptors, gene expression, uterine and bone tissue of ovariectomised rats [52]
Three RCTs investigate clinical effectiveness for Vitex 1. Lowers prolactin due to dopaminerg agnus-castus for oligo/amenorrhoea and PCOS effects [38-41,63]

$[61,62,64]$. One RCT demonstrated equivalence for

Bromocriptine and Vitex agnus-castus [63].

2. No change for serum prolactin [64]

3. FSH no change [39]

4. LH no change [39]

5. LH lowered [49]

6. Binds to $\beta$ oestrogen receptors $[38,43,69]$

7. Increased serum oestradiol $[49,64]$

8. Increased serum progesterone $[49,62]$

9. Improved pregnancy rates $[61,62]$

Three RCTs demonstrate positive fertility effects for Cimicifuga racemosa in women with PCOS $[65,67,68]$

Binds with a oestrogen receptors [44] in the pituitary and reduces $\mathrm{LH}$ secretion $[45,52,68]$

2. Increases luteal progesterone concentration $[65,67,68]$

3. Improves endometrial thickness for infertile women with PCOS [65,67,68]

4. Lowers $\mathrm{LH}$ in women with PCOS $[65,67,68]$

5. Improves FSH:LH ratio for women with PCOS [67]

6. Limits anti-oestrogen effects when used in combination with Clomiphene citrate for women with PCOS $[65,68]$ 
Table 1 Summary of evidence for the reproductive endocrinological effects of six herbal medicines in oligo/amenorrhoea, hyperandrogenism and PCOS

\section{(Continued)}

\begin{tabular}{lll}
\hline Cinnamon cassia & One animal study compared the effectiveness of & One pilot RCT demonstrated positive effects for \\
Aqueous extract (animal study) & Cinnamomum cassia with metformin against controls in rats & metabolic parameter's (HOMO and QUICKI) for \\
with PCOS. Hormone concentration was measured at 15 & Cinnamomum cassia in overweight women with \\
Ethanol extraction (Human & and 30 days [48] & PCOS [66]
\end{tabular}
trial)

with PCOS. Hormone concentration was measured at 15 PCOS [66]
1. Equivalence for metformin for reduced testosterone in PCOS [48]

2. Equivalence for metformin for reduced LH in PCOS [48]

3. Equivalence for metformin for reduced LH in PCOS [48]

4 Equivalence for metformin for reduced insulin resistance [48]

5. Improved metabolic profile for overweight women with PCOS [66]

\section{Herbal medicine}

Evidence

Botanic name

Pre-clinical in vitro and in vivo

Herbal extract

Tribulus terrestris

Ethanol extracts Three animal studies investigated the effects of Tribulus
terrestris, two for polycystic ovaries and one on oestrogen sensitive tissues in rats.

1. One study examined the oestrogenic effects of Tribulus terrestris on uterine and vaginal tissue of ovariectomised rats [51].

2. Two studies investigated the ovulation rates, number of corpus luteum and follicle characteristics in rats with polycystic ovaries following exposure to various doses of Tribulus terrestris $[46,47]$

Glycyrrhiza glabra (European liquorice)

Glycyrrhiza uralensis (Chinese liquorice)

Ethanol extract

Aqueous extract used in two pre-clinical studies
Two preclinical studies investigated the effects of Glycyrrhiza spp. for steroid hormone concentration and in polycystic ovaries.

1. Steroid hormone concentration in sterilised and oophrectomised rats following exposure to Glycyrrhiza spp. (kanzo) [53].

2. Morphological features of polycystic ovaries of rats following exposure to two Chinese herbal compounds with only Glycyrrhiza spp. as a common ingredient [50]
Data from clinical studies (non RCTs)

\section{Physiological effects in oligo/} amenorrhoea, hyperandrogenism and/or PCOS

\section{Ovulation induction in polycystic}

ovaries $[46,47]$

1. Healthy women $\mathrm{n}=8$ early menstrual cycle (follicular phase) Pre and post serum hormone concentration for FSH, LH testosterone and oestradiol at 8 am and $12 \mathrm{pm}$. Intervention consisted of Tribulus Terrestris $250 \mathrm{mg}$ per day over five days. Results showed significant increase in FSH and rise in LH (not significant) an increase in oestradiol and no change in testosterone concentration [56]

2. No oestrogenic effects in female reproductive tissues [51].

3. Increased FSH in healthy women [56]

4. Equivalence for ovulation induction for Tribulus Terrestris and Clomiphene for women with oligo/anovular infertility [60].

2. Equivalence of Tribulus terrestris and three ovulation in women with oligo/anovular infertility $(n=148)[60]$.

\section{Two clinical trials}

1. Single arm clinical trial investigating serum androgen concentration in healthy women aged 22-26, $(n=9)$ following administration of Glycyrrhiza spp. 7grams per day [55].

2. Single arm clinical trial including women with PCOS $(n=32)$ taking Spirinolactone [54]. ovulation induction pharmaceuticals evaluated
1. Increased aromatisation of testosterone to 17 beta oestradiol shown by significantly dose dependent reduced testosterone and increased oestradiol [53].

2. Reduced free and total testosterone [53].

3. Reduced serum androgens in healthy women [55].

4. Reduced androgen flare for women with PCOS using the anti-androgen pharmaceutical Spirinolactone [54].

5. Improved ovulation rates in polycystic ovaries [50]. 
Table 1 Summary of evidence for the reproductive endocrinological effects of six herbal medicines in oligo/amenorrhoea, hyperandrogenism and PCOS

\section{(Continued)}

Paeonia lactiflora in

combination with Glycyrrhiza

spp. Aqueous extract

Shakuyaku- kanzo-to (TJ-68)

One laboratory study examined the effects for the combination

Paeonia lactiflora and Glycyrrhiza uralensis on testosterone, oestradiol

FSH and LH in sterilised female rats [53].

Paeonia lactiflora in

combination with $\quad$ investigated for steroid hormonal effects on cultured human

Paeonia lactiflora and Cinnamomum cassia combination was

extract Unkei-to

incubated with different doses for 48 hours [42]
Two single arm clinical trials examined androgen concentrations Following treatment with Paeonia lactiflora and Glycyrrhiza uralensis in the Chinese herbal combination Shakuyaku-kanzo-to. One included infertile oligomenorrhoeic women with hyperandrogenism $(n=8)[58]$ and the other included women with oligo/amenorrhoea and $\operatorname{PCOS}(n=34)[59]$.

One clinical trial investigated the effects of Paeonia lactiflora and Cinnamomum cassia combination (Unkei-to) [57]. This single arm study included amenorrheic women aged $17-29$ years $(n=157)$ with a sub group of women with hyper-function. with a sub group of women with hyper functioning oligo/amenorrhoea $(n=42)$. Ovulation occurred in $61.3 \%$ of primary amenormeic women and in $27.3 \%$ of secondary amenorrheic women following two months of treatment [57].
1. Reduced total and free testosterone $[53,58,59]$

2. Increaed SHBG [59].

3. Reduced LH [53].

4. Reduced LH:FSH ratio [59].

5. Oestradiol slight increase (not significant) [53].

6. Improved ovulation in women with PCOS [58].

1. Increased granulosa production of oestradiol [42].

2. Increased granulosa production of progesterone [42].

3. Reduced LH in oligo/amenorrhoea [57].

4. Improved ovulation rates in oligo/ amenorrhoea [57]. 


\begin{tabular}{|c|c|c|c|}
\hline $\begin{array}{l}\text { Author and year } \\
\text { of publication }\end{array}$ & $\begin{array}{l}\text { Study design } \\
\text { and duration }\end{array}$ & Subjects & Intervention \\
\hline \multirow[t]{2}{*}{ Kilicdag [63] } & $\begin{array}{l}\text { Randomised comparative } \\
\text { effectiveness trial. } \\
\text { Treatment for } 3 \text { months. }\end{array}$ & $\begin{array}{l}\text { Eighty women, } 40 \text { with } \\
\text { hyperprolactin-aemia, } \\
40 \text { with cyclical mastalgia. }\end{array}$ & $\begin{array}{l}\text { Herbal extract Vitex agnus-castus } \\
40 \mathrm{mg} \text { in the commercial } \\
\text { preparation Agnucaston }{ }^{\oplus} \text { by } \\
\text { Biomeks, Germany. }\end{array}$ \\
\hline & & & $\begin{array}{l}1 \text { tablet per day. Bromocriptine } \\
\text { in the form of Parlodel produced } \\
\text { by Novartis, Turkey, } 2.5 \text { mg twice } \\
\text { daily. }\end{array}$ \\
\hline
\end{tabular}

Outcome measures

Comparison of difference between Vitex agnus-castus and Bromocriptine for serum prolactin concentration on days $5-8$ of the menstrual $628.5 \mathrm{mlU} /$

Vitex agnus-castus 32.4 mg/d in the commercial preparation Mastodynon liquid extract produced by Bionorica,

$$
\text { Germany. }
$$

fertility disorders and at 2 years

Secondary amenorrhoea, $\mathrm{n}=38$; luteal insufficiency, $n=31$; idiopathic infertility $n=27$.

Bergmann

Randomised, placebo controlled double blind study. Three months or

3 menstrual cycles.
Women with fertility disorders, $(n=67)$. Two sub-groups. $\begin{aligned} & \text { 1.oligomenorrhoea } \\ & n=37\end{aligned}$ herbal extracts of Caulophyllum in Mastodynon affect prolactin
30 drops per day over 3 months Mastodynon ${ }^{\circledR}$ additionally contain thalictroides, Lilium majus, Cyclamen Ignatia and Iris.

No evidence that therapeutic agents additional to $V$. agnus-castus concentration.

Herbal extract Phyto-Hypophyson ${ }^{\oplus}$ Primary outcome for by Steril-Pharma GmbH Herrsching, participants with Germany; contains Vitex agnus-castus amenorrhoea: at least plus Chelledonium majus and Silybum marion m (St Man's thiste) in homeopathic form. Additional herbal extracts have reported activity in hepatic function. There are no reports for direct reproductive effects. 150 drops per day ( $7.5 \mathrm{ml}$ per day).
Spontaneous menstruation, luteal phase length, serum hormone concentrations and pregnancy rates.

Hormonal data from 32 cases. In the third treatment month 66 complete data sets were available.

Reasons were as follows 4 due to drug reactions and 15 due to pregnancy reasons. one spontan: For progesterone $<1 \mathrm{ng} / \mathrm{mL}$ an increase to $>5 \mathrm{ng} / \mathrm{m}$ at the end of 3rd cycle

For oligomenorrhoea: Shortened menstrual cycle of at least 4 days Earlier ovulation of at least 3 days. For anovulatory oligomenorrhoea: Mid luteal progesterone increase ( $>50 \%$ 5-10 days before menstruation. Secondary clinical outcomes, pregnancy rates and take home baby rates.

\section{Results and level of significance}

Mean prolactin concentration before and after in the V.agnus-castus arm $946 \mathrm{mlU} / \mathrm{L}( \pm 173.5)$ to $529 \mathrm{mlU} / \mathrm{l}( \pm 279.7)$ $\mathrm{p}<0.0001$. In the Bromocriptine arm; $885.0 \mathrm{mlU} /( \pm 177.5)$ to $472.68 \mathrm{mlU} /$ $( \pm 265.6), p<0.0001$

Equivalence demonstrated for the significant reduction of serum prolactin for $V$. agnus-castus and Bromocriptine $(P=0.96)$.

Non-significant improvement in clinical parameters in $57.6 \%$ of women in treatment group versus $36.0 \%$ in placebo group, $\mathrm{P}=0.069$. In a subgroup of women with luteal insufficiency $(n=21)$ there were significant improvements in clinical parameters in the treatment group compared to placebo $(p=0023)$. 15 women conceived in the treatment group compared to 8 in placebo group in the first 3 months (while women were treated).

All pregnant women were withdrawn from the study. 4 women had miscarriags, at in the a hive arm. After 2 years there were 21 more pregnancies with 2 miscariages spread over active and placebo groups.

Oligomenorrhoeic subgroup - clinical outcomes were significantly improved in the treatment arm at $82 \%$ compared to $45 \%$ in placebo arm $P=0.021$. When the amenorrheic group were included in analysis, differences were not significant $p=0.19$.

Mid luteal progesterone concentration in oligomenorrhoeic sub-group was significantly higher than the placebo group $p=0.0479$

At 6 months following conclusion of treatment, the take home baby rate with treatment was $18.7 \%$ compared to $6.4 \%$ in placebo group. Not statistically significant.

\section{Comments}

All participants completed the trial. Adverse reactions; zero eported in V. agnus-castus group; $12.5 \%$ of participants eported adverse reactions in the Bromocriptine grou

Small sample sizes with groups. Insufficiently powered groups. Insuficiently powered to correctly identify the effects: ( $\pm 5 \%$, $95 \%$ confidence).

Numbers too small for statistica significance in clinical outcomes Preparation 'Mastodynon' contains $\checkmark$ agnus-castus plus other herbal extracts which may have

confounded outcome measures.

Inconsistencies in data assessment include the recommendation for reatment with Mastodynon over $3-6$ months yet it was tested for 3 months.

Women with infertility were included in this study however data from women who conceived were excluded. This may have led to an underestimation of treatment effect (type 1 error).

Diagnosis for anovulatory amenorrhoea is not well described. Non-statistically significant take home baby ates were complicated by insufficient sample size. 366 patients are required to have a $95 \%$ chance, as significan at the $5 \%$ level, an increase in take home baby rates from $6 \%$ in the placebo group to $18 \%$ in the experimental group. The authors conclude that this preparation may be useful if given 3-6 months, yet they only tested for 3 months. 
Table 2 Summary of randomised controlled trials for five herbal medicines in oligo/amenorrhoea, hyperandrogenism and PCOS (Continued)

\begin{tabular}{|c|c|c|c|}
\hline $\begin{array}{l}\text { Milewicz A, } \\
\text { Gejdel E, } \\
\text { et al. [64] }\end{array}$ & $\begin{array}{l}\text { Randomised placebo } \\
\text { controlled, double blind, } \\
\text { trial. Three months. }\end{array}$ & $\begin{array}{l}52 \text { women with latent } \\
\text { hyperprolactinaemia and } \\
\text { luteal phase defects. } \\
\text { Participants stratified for } \\
\text { cycle length, height }(\mathrm{cm}) \\
\text { and weight (kgs) and } \\
\text { randomised. Baseline } \\
\text { differences between } \\
\text { arms were not significant } \\
p=0.63, p=0.48 \text { and } \\
p=0.37 \text { respectively. } 37 \\
\text { complete case reports: } \\
\text { Treatment arm } n=17 \text {, } \\
\text { placebo } n=20 \text {. }\end{array}$ & $\begin{array}{l}\text { Vitex agnus-castus extract } 20 \mathrm{mg} \\
\text { in the commercial preparation of } \\
\text { Strotan }{ }^{\circledR} \text { Hersteller: Pharma } \\
\text { Stroschein GmbH, Hamburg, } \\
\text { Germany. } 1 \text { capsule per day or } \\
\text { placebo. }\end{array}$ \\
\hline
\end{tabular}
Serom 30 minutes following intra venous TRH (200mcg) stimulation Luteal phase length Luteal phase before and of number of days Measurements trealme on menstuas co days 5 to $10.5( \pm 43)(p<0.005)$. plo to ond 20 for FSH 1 H oestradiol 3.5 ( \pm 4.3$)(0<0.005$ ), placebo progesterone, DHEAs, thyroid, 3.4 progesterone, DHEAs, luteal (day 20) serum 政, progesterone concentration before and after; treatment arm $2.46( \pm 0.70)$ to 9.69 $( \pm 6.34), p<0.001$. Placebo $1.99( \pm 0.65)$ to $2.34( \pm 0.59)$ $\mathrm{p}=0.08$.

Mid-cycle oestradiol; treatment arm $131.6( \pm 25.0)$ to $151.6( \pm 25.4), p<0.05$ $131.6( \pm 25.0)$ to $151.6( \pm 25.4), p<0.05$
Placebo: $119.5( \pm 26.0)$ to $131.1( \pm 33.2)$ $p=0.22$. Pregnancies in treatment group $n=2$.

In this study 52 women were eligible to participate, statistical analyses were performed on

data from 37 women.

There is missing data due to the presence of luteinised

unruptured follicles (9 women) These data were not included in analyses. Six women did not present for further investigation No description of the distribution of drop-outs or missing data. This suggests the potential imbalance between intervention and control and a possible overexaggeration for treatment effect. Intention to treat analysis was not performed.

Randomised controlled 147 women aged less trial using with an active control arm for comparative effectiveness. One menstrual cycle. un-explained infertility and recurrent clomiphen resistance for ovulation participants were excluded $(n=28)$. Anovulation was diagnosed by serum oestradiol < $200 \mathrm{ng} / \mathrm{ml}$ and absence of a dominant ovarian follicle on day 9 of the menstrual cycle Complete data sets available for 119 women

All women received Clomiphene citrate (clomiphene) $150 \mathrm{mg}$ on menstrual cycle days 3-7. A randomised group also took Cimicifuga racemosa $20 \mathrm{mg}$ per day between days 1-12. Cimicifuga racemosa described as phytoestrogens' was provided in the commercial preparation Klimadynon, manufactured by Norica in Germany. A trigger injection (human chorionic gonadotropin, 10000 IU) and timed intercourse was recommended when a dominant follicle $>17 \mathrm{~mm}$ was observed.
Pregnancy rate measured as increasing serum human chorionic gonadotropin HCG) over two days. Clinica pregnancy defined as wection of gestational sac with embryonic heart-beat. Endometrial thickness measured by ultrasoun concurrent with follicle maturation monitoring. Number of days to ovulation (trigger injection) serum concentration for FSH oestradiol and LH. Luteal progesterone measured on days 21-23 of the menstrua cycle Miscarriage and multiple pregnancy rates
Pregnancy rate in clomiphene alone group was $20.3 \%$ and $43.3 \%$ in the clomiphene plus Cimicifuga racemosa group $(P<0.01)$. Clinical pregnancy rate in the combination group was $367 \%$ versus $13.6 \%$ in the clomiphen alone group $(P<0.01)$. Endometrial thickness in combination group was $8.9( \pm 1.4)$ versus $7.5( \pm 1.3)(p<0.001)$ Days to ovulation in clomiphene alone group was $13.0 \pm 1.1$ and in the clomiphene plus Cimicifuga racemosa group $14.2 \pm 1.3$ (n.s.). Lutea progesterone peak $(\mathrm{ng} / \mathrm{ml})$ in combination group was $13.3( \pm 3.1)$ versus $9.3( \pm 2.0)$ in clomiphene alone group $(p<0.01)$. All other hormone measures were not significantly differen
Unaccounted confounding factors include medications, fertility status, duration of latent hyperprolactinaemia. No detailed current baseline cinterla for other causes of infertility. Confounding factors include current male fertility status. This may have caused an imbalance between the two groups. There is no description of the distribution of excluded (anovulatory) participants between groups. 
Table 2 Summary of randomised controlled trials for five herbal medicines in oligo/amenorrhoea, hyperandrogenism and PCOS (Continued)

Kamel [67] Randomised controlled Women aged 21-27 with Cimicifuga racemosa extract Sorum measurements during Positive outcomes for Cimicifuga

trial with an active control primary or secondany Cimicifuga racemosa extrac

group. Comparative infertility. Diagnosis of Neumarkt i.d. OBF Germany

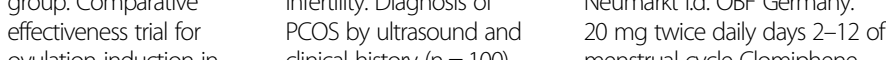

effectiveness trial for $\quad$ PCOS by ultrasound and

ovulation induction in clinical history $(n=100)$.

menstrual cycles.

citrate (clomiphene) $100 \mathrm{mg}$

one $(n=50)$ received

Clomiphene citrate

$100 \mathrm{mg}$ days $2-7$ of the

cycle. Trigger injection (Human

and timed intercourse

menstrual cycle; group recommended when dominan

two $(n=50)$ received

follicle (>18 mm) was observed

for days $2-12$ of

menstrual cycle.

Non-blinded randomised Women with PCOS and

controlled trial. infertility, $n=194$

All participants received

pharmaceutical ovulation

primary and secondary induction (Clomiphene citrate

infertility and duration of

infertility (months).

Treatment arm $n=96$,

control $n=98$.

$150 \mathrm{mg}$ on days 3-7 of cycle);

trigger injection (HCG 10000 IU

Pregnyl), timed intercourse and

progesterone support (oral

Randomisation for 206

women 12 were excluded

due to failure to respond

control $n=5$ ). took Cimicifuga racemosa $120 \mathrm{mg}$

per day (Klimadynon ${ }^{\oplus}$ ) follicular phase for FSH, LH and

racemosatcomes for Cimicifuga

FSH:LH ratio. Mid luteal

for reduced day 2-5: $1 \mathrm{H}(\mathrm{p}=0.007)$

progesterone. Ultrasound

and improved FSH to LH ratio

observation of endometrial $\quad(p=0.06)$, mid luteal progesterone

thickness Pregnancy rates

including twin preg rates

including twin pregnancies.

$(p=0.0001)$, endometrial thickness

$(p=0.0004)$. Pregnancy rates were

hyperstimulation.

higher in the Cimicifuga racemosa

No detail for diagnostic criteria for PCOS. Confounding fertility factors not described. Drop-out reasons were not reported seven in Cimicifuga racemosa group and four in rlomiphe group
group.

Primary outcomes pregnanc rates. Secondary outcomes:

1. Number of days to ovulation (trigger injection). Follicular maturation monitored by ultrasound

2. Endometrial thickness monitored by

ultrasound.

3. Serum hormones during

follicular phase oestradiol,

LH and FSH. Luteal

progesterone measured

day 21-23 of the cycle.

4. Pregnangy outcom

for early miscarriage

not statistically significant $(p=01)$. (34.8\%) for the clomiphene plus

$12.9( \pm 2.3)$ in the clomiphen
Adverse events (4 women) and twin

pregnancy's (two women) were

not significantly different between

groups.

Pregnancy rates were 33 out of 192 cycles (17.2\%) for the clomiphene alone group and 71 out of 204 cycles

Cimicifuga racemosa group.

Number of days to trigger

injection was $15( \pm 1.7)$ for the

clomiphene alone group and

$12.0( \pm 1.9)$ in the clomiphene

plus Cimicifuga racemosa

group $(p=0.01)$

Endometrial thickness in the

clomiphene alone group was

$8.5 \mathrm{~mm}( \pm 1.9)$ compared to

plus Cimicifuga racemosa

group $(p<0.001)$

Serum LH was $8.0( \pm 0.9)$ in the

clomiphene group and 5.7

$\pm 0.9)$ in the clomiphene plus

Cimicifuga racemosa group

$(p<0.001)$ and oestradiol was

$2283(+30.2)$ in the clomiphen

alone group and 2995 ( \pm 38.9 ) Vin

alone group and $299.5( \pm 38.9) \backslash$ in
the clomiphene plus Cimicifuga

the clomiphene plus Cim

racemosa group $(p=0.01)$

Miscarriages were 5 out of 192

cycles in the clomiphene group

and 6 out of 204 cycles in the

clomiphene plus Cimicituga
racemosa group (n.s.).
Non-blinding compromised the internal validity of the findings in this study. Confounding variables and clinicians attitudes and may have led to differences which were unaccounted for between the two groups. However the outcomes are objective with a statistically

powered sample size.

Measures for miscarriages are based on per cycle are not valid. Miscarriages per pregnancy are of greater relevance.

The miscarriage rate per pregnancy for the clomiphene alone group was 5 out of $33(15.2 \%)$ and 6 out of $71(8.5 \%)$ in the clomiphene plus Cimicifuga racemosa group 
Table 2 Summary of randomised controlled trials for five herbal medicines in oligo/amenorrhoea, hyperandrogenism and PCOS (Continued)

\begin{tabular}{|c|c|c|c|c|c|c|}
\hline $\begin{array}{l}\text { Wang et al. } \\
2008 \text { [66] }\end{array}$ & $\begin{array}{l}\text { Double blinded, placebo } \\
\text { controlled randomised } \\
\text { trial (pilot). Eight weeks. }\end{array}$ & $\begin{array}{l}15 \text { overweight women } \\
\text { with oligo/amenorrhoea } \\
\text { and polycystic ovaries on } \\
\text { ultrasound. Mean body } \\
\text { mass index } 28.8 \pm 1.3 \mathrm{~kg} / \mathrm{m} 2 \text {. } \\
\text { Mean age } 31.1 \pm 2.0 \text { years }\end{array}$ & $\begin{array}{l}\text { Cinnamomum cassia extract } 333 \mathrm{mg} \\
\text { (Integrity Nutraceuticals International } \\
\text { Sarasota, Florida) or placebo. One } \\
\text { tablet three times per day. }\end{array}$ & $\begin{array}{l}\text { Primary outcomes: Insulin } \\
\text { resistance and sensitivity. } \\
\text { Secondary outcomes } \\
\text { oestradiol and testosterone } \\
\text { concentration. Body mass } \\
\text { index (BMI). Before and after } \\
\text { treatment comparisons } \\
\text { between randomised groups } \\
\text { plus comparison between } \\
\text { treatment group and normal } \\
\text { ovulatory, normal weight } \\
\text { women. Adverse events. }\end{array}$ & $\begin{array}{l}\text { Improved insulin sensitivity } \\
\text { (QUICKI) in the treatment } \\
\text { group. } 0.35 \text { to } 0.38,(7.7 \%) \\
\text { p }<0.03 \text {. Insulin resistance } \\
\text { (HOMO-IR) significantly } \\
\text { reduced in treatment group } \\
2.57 \text { to } 1.43(44.5 \%) p<0.03 \text {. } \\
\text { Controls no change insulin } \\
\text { sensitivity or insulin resistance. } \\
\text { No change in either group for } \\
\text { BMl, testosterone and oestradiol. } \\
\text { Differences between Cinnamomum } \\
\text { cassia group and normal weight } \\
\text { and ovulatory controls were not } \\
\text { significant. }(\mathrm{P}<0.17) \text {. No reported } \\
\text { adverse reactions. }\end{array}$ & $\begin{array}{l}\text { Small pilot study, the authors report } \\
\text { that larger studies are required to } \\
\text { confirm findings. Small sample size } \\
\text { may explain non-significant } \\
\text { comparison with normal weight } \\
\text { and ovulating women. } \\
\text { Reproductive outcomes were } \\
\text { unchanged in this study however } \\
\text { the duration of the study was } \\
\text { insufficient to demonstrate } \\
\text { reproductive changes. }\end{array}$ \\
\hline
\end{tabular}




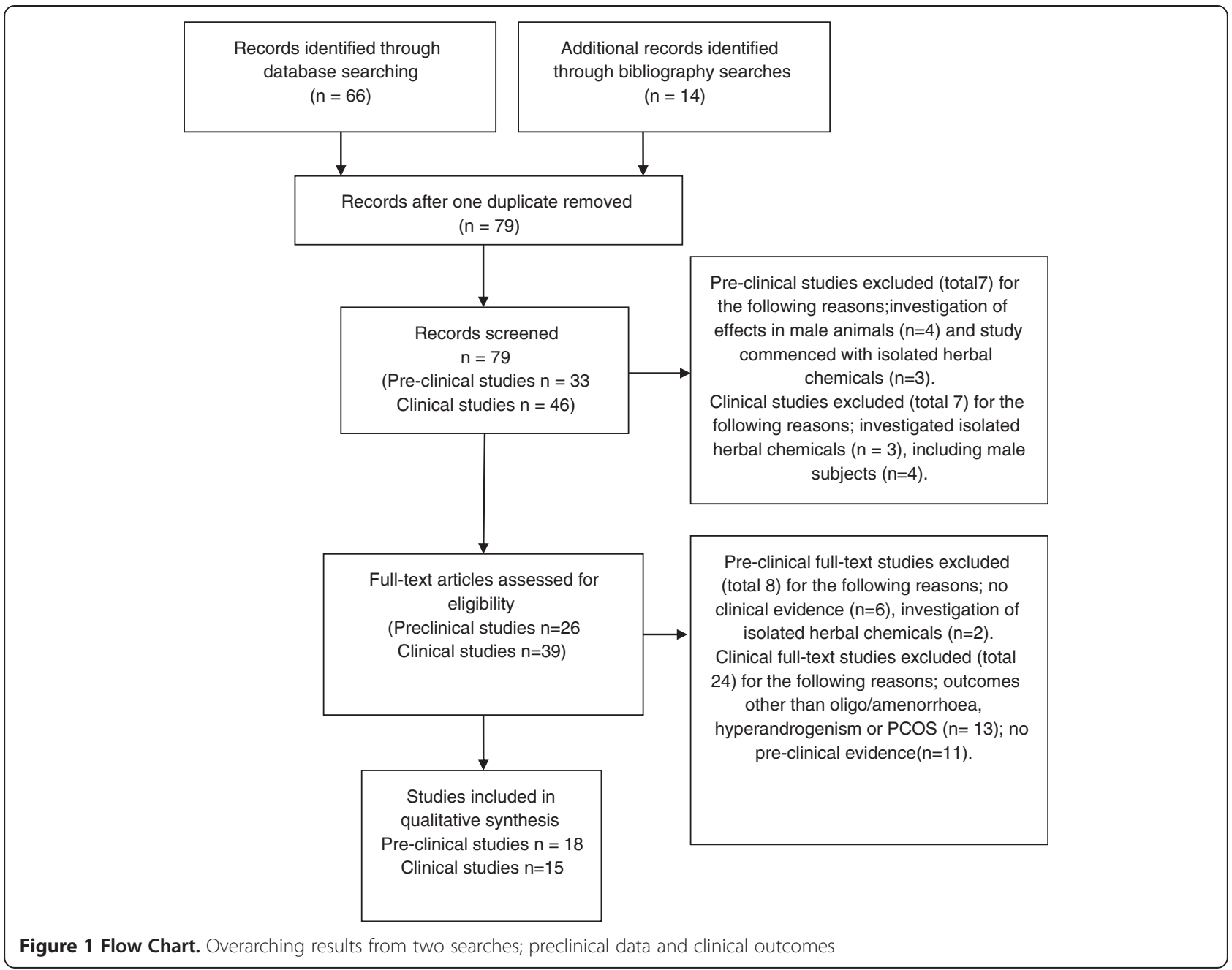

isolated herbal chemicals $(\mathrm{n}=3)$; inclusion of male subjects $(n=4)$; no pre-clinical evidence $(n=11)$ and conditions different to those specified $(\mathrm{n}=13)$.

\section{Excluded studies}

Details of excluded studies investigating herbal medicines with clinical evidence but no preclinical evidence were provided in Table 3. Herbal medicines with preclinical evidence but no clinical evidence were provided in Table 4, and investigations into isolated chemicals derived from herbal medicine were presented in Table 5 .

Seven RCTs examined commercially produced herbal medicine extracts. These were Vitex agnuscastus in the form of Strontan ${ }^{\circ}[64]$, Mastodynon ${ }^{\circ}$ [61], Phyto Hypophyson [62] and Agnacaston ${ }^{\circ}[63]$ and Cimicifuga racemosa in the form of Klimadynon ${ }^{\circ}$ $[65,67,68]$ (Table 2).
Herbal medicines with effects in oligo/amenorrhoea, hyperandrogenism and PCOS

The results of preclinical studies and clinical studies have been summarised together for each of the six herbal medicines.

\section{Vitex agnus-castus}

Pre-clinical and clinical evidence was found for Vitex agnus-castus for lowered prolactin, improved menstrual regularity and treatment of infertility. Vitex agnus-castus contains a variety of compounds which bind to dopamine type 2 (DA-2) receptors in the brain; reduce cyclic adenosine mono phosphate (cAMP) and lowered prolactin secretion (Table 1). This was demonstrated in studies using recombinant DA-2 receptor proteins, and basal and stimulated rat pituitary cell cultures [38-41]. Prolactin lowering effects were found in normal and ovariectomised rats [49]. Additional agonistic opiate effects were 
Table 3 Herbal medicines with clinical evidence not included in this review

\begin{tabular}{|c|c|c|}
\hline Herbal medicine & $\begin{array}{l}\text { Clinical evidence (or potential) for PCOS and associated } \\
\text { oligo/amenorrhoea or hyperandrogenism }\end{array}$ & $\begin{array}{l}\text { Reason for non-inclusion - insufficient pre-clinical evidence } \\
\text { for mechanism of effects for whole herbal extract }\end{array}$ \\
\hline $\begin{array}{l}\text { Camellia sinensis } \\
\text { (green tea) }\end{array}$ & Hormone concentration in obese women with PCOS [71]. & $\begin{array}{l}\text { Isolated constituent (epigallocatechin gallate 1) examined } \\
\text { [72]. No evidence found for effects for whole herbal extract } \\
\text { in PCOS, oligo/amenorrhoea and hyperandrogenism. }\end{array}$ \\
\hline $\begin{array}{l}\text { Mentha spicata } \\
\text { (spearmint tea) }\end{array}$ & Lowered testosterone in women with PCOS $[73,74]$. & $\begin{array}{l}\text { No evidence for mechanism of effect found for PCOS, oligo/ } \\
\text { amenorrhoea or hyperandrogenism. }\end{array}$ \\
\hline $\begin{array}{l}\text { Ginkgo Biloba } \\
\text { (ginkgo) }\end{array}$ & Metabolic hormone management for type two diabetes [75]. & $\begin{array}{l}\text { No evidence for mechanism of effect in PCOS, oligo/ } \\
\text { amenorrhoea or hyperandrogenism found. }\end{array}$ \\
\hline $\begin{array}{l}\text { Grifola frondosa } \\
\text { (miatake } \\
\text { mushroom) }\end{array}$ & Ovulation rates in PCOS [76]. & $\begin{array}{l}\text { No evidence for mechanism of effect in PCOS, oligo/ } \\
\text { amenorrhoea or PCOS revealed. }\end{array}$ \\
\hline $\begin{array}{l}\text { Linum } \\
\text { usitatissimum (flax } \\
\text { seed) }\end{array}$ & $\begin{array}{l}\text { Menstrual regulation }[77,78] \text { and hormonal concentration }[78-80] \\
\text { in post-menopausal women. }\end{array}$ & $\begin{array}{l}\text { No mechanism of effect in PCOS, oligo/amenorrhoea or } \\
\text { hyperandrogenism found. }\end{array}$ \\
\hline $\begin{array}{l}\text { Pygeum africanum } \\
\text { (pygeum) }\end{array}$ & Anti-androgen effects in prostatic hypertrophy [81]. & $\begin{array}{l}\text { No evidence for mechanism of effect found in PCOS, oligo/ } \\
\text { amenorrhoea or hyperandrogenism (in female cell cultures } \\
\text { or animals). }\end{array}$ \\
\hline $\begin{array}{l}\text { Serrenoa repens } \\
\text { (saw palmetto) }\end{array}$ & Anti-androgen effects in chronic pelvic pain and prostatitis [82-84]. & $\begin{array}{l}\text { No mechanism of effect in PCOS, oligo/amenorrhoea or } \\
\text { hyperandrogenism (in female cell cultures or animals). }\end{array}$ \\
\hline $\begin{array}{l}\text { Silybum marianum } \\
\text { (St Mary's thistle) }\end{array}$ & Fatty liver disease in type two diabetes [85]. & $\begin{array}{l}\text { No mechanism of effect in PCOS, oligo/amenorrhoea or } \\
\text { hyperandrogenism. }\end{array}$ \\
\hline $\begin{array}{l}\text { Stachys } \\
\text { lavandulifolia } \\
\text { (wood betony) }\end{array}$ & $\begin{array}{l}\text { Evidence for improved uterine bleeding (including } \\
\text { oligomenorrhoea and amenorrhoea) in women with PCOS } \\
\text { comparable with Medroxyprogesterone acetate [86]. }\end{array}$ & $\begin{array}{l}\text { No mechanism of effect studies found for whole herbal } \\
\text { extract in PCOS and or associated oligo/amenorrhoea and } \\
\text { hyperandrogenism. }\end{array}$ \\
\hline $\begin{array}{l}\text { Urtica dioca } \\
\text { (nettle root) }\end{array}$ & Anti-androgen effects in women [87]. & $\begin{array}{l}\text { Anti-androgen effects through interaction with SHBG in } \\
\text { prostate cells [88-90]. Anti-inflammatory and anti-nociceptive } \\
\text { effects [91] No evidence for effects of Urtica dioca in female } \\
\text { cell cultures or animals. }\end{array}$ \\
\hline
\end{tabular}

Other excluded studies investigated the herbal medicines included in this review examining conditions other than PCOS, oligo/amenorrhoea and hyperandrogenism. These included investigations into effectiveness for Vitex agnus-castus for pre-menstrual syndrome [92-97] and mastalgia [98,99], Cimicifuga racemosa for menopausal symptoms [100] and Glycyrrhiza spp with Paeonia lactiflora libido in males [101].

observed in studies using human opiate receptors cell cultures [70].

Clinical equivalence for prolactin lowering effects of Vitex agnus-castus (Agnucaston ${ }^{\odot} 40 \mathrm{mg}$ per day) and the pharmaceutical Bromocriptine (Parlodel ${ }^{\bullet} 5 \mathrm{mg}$ per day) was found in one study including 40 women with hyperprolactinaemia [63]. Mean concentrations for prolactin following three months treatment with Vitex agnuscastus was significantly reduced from $946 \mathrm{mIU} / \mathrm{l}( \pm 173)$ to $529 \mathrm{mIU} / \mathrm{l}( \pm 297)(\mathrm{p}<0.001)$. Comparatively, mean prolactin concentration in the Bromocriptine group was significantly reduced from $885 \mathrm{mIU} / \mathrm{l}( \pm 178)$ to $473 \mathrm{mIU} / \mathrm{l}$ $( \pm 266)(\mathrm{p}<0.001)$ demonstrating that both treatments were effective treatment for women with hyperprolactinaemia (normal reference range 25-628 mIU/l). The mean difference in prolactin reduction of the two groups was not significant $(\mathrm{p}=0.96)$ (Table 2$)$.

Positive effects for Vitex agnus-castus in oligo/amenorrhoea and infertility was demonstrated in three placebo controlled RCTs $[61,62,64]$. In a study including

Table 4 Herbal medicines with pre-clinical evidence not included in this review

\begin{tabular}{lll}
\hline Herbal medicine & $\begin{array}{l}\text { Pre-clinical evidence for (potential) effects in reproductive endocrinology in } \\
\text { PCOS and associated oligo/amenorrhoea and hyperandrogenism }\end{array}$ & Reason for exclusion \\
\hline $\begin{array}{l}\text { Curcuma longa } \\
\text { (turmeric) }\end{array}$ & Anti-androgen effects [102]. & $\begin{array}{l}\text { No clinical evidence examining } \\
\text { effectiveness in women was found. }\end{array}$ \\
$\begin{array}{l}\text { Matricaria chamomilla } \\
\text { (Chamomile) }\end{array}$ & $\begin{array}{l}\text { Reduced luteinising hormone and improved ovarian morphology in animals with } \\
\text { PCOS [103]. }\end{array}$ & No clinical data found. \\
$\begin{array}{l}\text { Mentha piperita } \\
\text { (peppermint) }\end{array}$ & Anti-androgen effects in animals [104]. & No clinical data for women. \\
$\begin{array}{l}\text { Silybum marianum } \\
\text { (St Marys thistle) }\end{array}$ & Anti-proliferative antioxidant and biochemical effects in the liver [105]. & No clinical evidence including women \\
\hline
\end{tabular}

Studies investigating chemical compounds derived from the herbal medicines, included in this review but investigating different outcomes were found for Vitex agnus-castus [70] and Cimicifuga racemosa [106]. 


\begin{tabular}{|c|c|}
\hline Isolated chemicals & Evidence for effects \\
\hline Phytoestrogens & Hormonal effects in ovarian granulosa cells [107]. \\
\hline Berberine & $\begin{array}{l}\text { Comparison with metformin in PCOS [16]; } \\
\text { ovarian theca cell hormone production [108]. }\end{array}$ \\
\hline $\begin{array}{l}\text { Catechin derived } \\
\text { from Camellia sinensis }\end{array}$ & $\begin{array}{l}\text { Effects of epigallocatechin gallate } 1 \text { on cellular } \\
\text { metabolic endocrinology [72]. }\end{array}$ \\
\hline $\begin{array}{l}\text { Sapponins derived } \\
\text { from Tribulus terrestris }\end{array}$ & Effects on reproductive endocrinology [109]. \\
\hline $\begin{array}{l}\text { Paeoniflorin, } \\
\text { glycyrrhizin and } \\
\text { glycyrrhetic acid }\end{array}$ & Ovarian androgen production [110]. \\
\hline $\begin{array}{l}\text { Isoflavones isolated } \\
\text { from Vitex agnus- } \\
\text { castus }\end{array}$ & $\begin{array}{l}\text { Selective oestrogen receptor activity } \\
\text { (competitive inhibition via beta oestrogen } \\
\text { receptors) [36]. }\end{array}$ \\
\hline
\end{tabular}

women with menstrual irregularity and infertility $(\mathrm{n}=96)$, menstrual cyclicity was significantly improved for women treated with Vitex agnus-castus (Mastodynon ${ }^{\circ} 30$ drops per day for three months) compared to placebo $(\mathrm{p}=0.023)$ [61] (Table 2). Another study, including women with sub fertility $(n=67)$, showed improved menstrual cyclicity for a sub-group of women with oligomenorrhoea following treatment with Vitex agnuscastus (Phyto-Hypophyson $7.5 \mathrm{ml}$ per day) compared to placebo, $(\mathrm{p}=0.023)$ [62] (Table 2). A third study including women with hyperprolactinaemia $(\mathrm{n}=37)$ demonstrated improved menstrual cyclicity by an increased average number of luteal days from 3.4 days $( \pm 5.0)$ to 10.5 days $( \pm 4.3) \quad(\mathrm{p}<0.005)$ following treatment with Vitex agnus-castus (Strotan $20 \mathrm{mg}$ per day) for three months. The placebo group reported average number of days in the luteal phase was 3.4 $( \pm 5.1)$ at baseline and $5.5( \pm 5.2)$ at three months, which was not significant $(\mathrm{p}=0.22)$ [64] (Table 2). Methodological shortcomings included not reporting baseline characteristics for subgroups and small sample sizes; however clinical outcomes demonstrated physiological effects consistent with laboratory and animal findings (Tables 1 and 2).

\section{Cimicifuga racemosa}

Cimicifuga racemosa was found to lower LH in two laboratory studies both examining cell cultures from ovariectomised rats [45,52] (Table 1). The mechanism occurred through competitive inhibition of oestrogen following the selective binding of oestrogen receptors $(E R \alpha)$ on the hypothalamus and pituitary [52]. An earlier study found contrary results for reduction of LH, however this study investigated an isolated flavonoid and suggested that other constituents may be active [37].

Three RCTs corroborate the positive fertility effects for Cimicifuga racemosa in women with PCOS, used in conjunction and when compared with the pharmaceutical Clomiphene citrate (clomiphene), [65,68,71] (Table 2). Results were reported for 441 women and show improved pregnancy rates when Cimicifuga racemosa was added to clomiphene during one menstrual cycle. In a study including women with PCOS $(n=147)$, pregnancy rates for the group receiving combined therapy (clomiphene $150 \mathrm{mg}$ plus Cimicifuga racemosa $20 \mathrm{mg}$ per day (Klimadynon $\left.{ }^{\circ}\right)$ ) were $43.3 \%$ compared to $20.3 \%$ for women receiving only clomiphene [65] (Table 2). In another study using similar methodology $(n=100)$ pregnancy rates were $34.8 \%$ for the group treated with Cimicifuga racemosa plus clomiphene compared to $17.2 \%$ for women treated with clomiphene alone [68] (Table 2). Another study included women with PCOS and infertility $(\mathrm{n}=100)$ compared Cimicifuga racemosa (Klimadynon ${ }^{\circ}$ ) and clomiphene over three months for hormone concentrations and pregnancy rates. Pregnancy rates were higher in the women in taking Cimicifuga racemosa compared to clomiphene, $14 \%$ and $8 \%$ respectively; however differences were not statistically significant. This study found significant effects for lowered luteinising hormone for women with PCOS receiving Cimicifuga racemosa compared to clomiphene $(\mathrm{p}=0.007)$ [67]. Findings from clinical studies concur with laboratory and animal studies; however potential risks for bias include performance and collection bias due to lack of blinding (Table 2).

\section{Tribulus terrestris}

Two laboratory based RCT's examined the effects of Tribulus Terrestris in rats with polycystic ovaries induced with oestradiol valerate [46,47] (Table 1). Both studies demonstrate significantly improved ovulation rates for animals treated with two doses of Tribulus terrestris extracts compared to controls. Although the endocrinological effects were not described in either study, laboratory findings of ovulation induction are supported by the clinical findings of elevated FSH following treatment with Tribulus terrestris [56] (Table 2).

A prospective, observational clinical trial examined the endocrine effects of Tribulus terrestris $750 \mathrm{mg}$ per day, over five days in eight healthy women (aged 28-45). A significant increase in mean serum FSH concentration from $11 \mathrm{mIU} / \mathrm{ml}$ before treatment to $17.75 \mathrm{mIU} / \mathrm{ml}$ following treatment $(\mathrm{P}<0.001)$ was demonstrated. Pretreatment FSH levels returned following cessation of treatment (Table 1). Another clinical study evaluated the equivalence of Tribulus terrestris (Tribestan ${ }^{\circ}$ ) and pharmaceuticals for ovulation induction in women with oligo/anovular infertility $(\mathrm{n}=148)$, [60]. During the three month follow up, ovulation rates were highest with epimestrol (74\%), followed by Tribulus terrestris (60\%), clomiphene (47\%) and cyclofenil (24\%). However, the evidence for Tribulus terrestris should be interpreted 
with caution due to risks for bias in clinical studies. One study was uncontrolled with a small number of healthy participants [56], the second study did not report baseline characteristics, methods for allocation to treatment groups and data were not statistically analysed [60] (Table 1).

\section{Glycyrrhiza spp}

Androgen lowering effects for Glycyrrhiza spp. have been demonstrated in one laboratory study examining hormone concentration in female rats (Glycyrrhiza uralensis), [53] and corroborated in two clinical trials, one including healthy women [55] and the other including women with PCOS (Glycyrrhiza glabra) [54] (Table 1). The animal study reported significantly reduced free and total testosterone and increased oestradiol in sterilised rats and no hormonal changes in oophrectomised rats. The authors conclude that the hormonal effects occurred primarily in the ovary via enhanced aromatisation of testosterone to 17-beta oestradiol. The investigators also observed significantly increased oestradiol. There were no changes to FSH or LH in androgen sterilised or oophrectomised rats [53].

Another animal study examined the effects of Glycyrrhiza uralensis on the morphological features of polycystic ovaries using immunohistochemistry [50] (Table 1). This study demonstrated significantly increased ovulation rates by the number of corpus luteum in polycystic ovaries compared with controls. The authors propose that the mechanism of effect for Glycyrrhiza uralensis was competitive inhibition of oestrogen at oestrogen receptor sites, limiting the production of nerve growth factor (NGF), its neurotropic effects and inhibition of sympathetic neurological involvement in the pathogenesis of polycystic ovaries.

Two clinical studies examined the androgen lowering effects of Glycyrrhiza Glabra. A single arm clinical trial demonstrated reduced testosterone in healthy women aged $22-26$ years $(n=9)$ over two menstrual cycles. Treatment with Glycyrrhiza glabra, 7 grams per day reduced testosterone from 27.8( \pm 8.2$)$ to $17.5( \pm 6.4), \mathrm{p}<0.05$ [55]. Another single arm clinical trial investigated the effects of Glycyrrhiza glabra in women with PCOS, $(\mathrm{n}=32)$. Glycyrrhiza glabra $3.5 \mathrm{~g}$ per day was added to anti-androgen pharmaceutical treatment, Spirinolactone $100 \mathrm{mg} /$ day over two menstrual cycles. An unwanted side effect for Spirinolactone was the flare of androgens during the initial phase of treatment. This study demonstrated reduced concentrations of testosterone during the first four days of treatment at $103 \pm 29 \mathrm{ng} / \mathrm{d}$ in the Spirinolactone group compared to $91 \mathrm{ng} / \mathrm{d}( \pm 19)$ when combined with Glycyrrhiza glabra $(\mathrm{p}<0.05)$ [54] (Table 1). Consistent laboratory and clinical outcomes were demonstrated however limitations included design shortcomings. Both clinical studies were open label observational design with small sample sizes; one included healthy participants. Rigorous studies are needed to confirm the androgen lowering effects of Glycyrrhiza spp. in hyperandrogenism and PCOS.

Results for Glycyrrhiza Spp. (and indeed any herbal ingredient) were complicated in this case by the variation in herbal extraction processes and subsequent variability in chemical profiles of the herbal ingredients. The laboratory studies of the herbal material were based on aqueous extracts of crude material whilst the clinical studies were based on ethanol extracts. Despite variability in the herbal extraction methods, both laboratory and clinical studies demonstrated anti-androgenic effects.

\section{Paeonia lactiflora and glycyrrhiza uralensis}

One laboratory study and two clinical investigations provided evidence for the two herb combination, Glycyrrhiza uralensis and Paeonia lactiflora [53,58,59] (Table 1). An animal study found significant reductions in free and total testosterone following exposure to the combination [53] (Table 1). These findings were supported in two open label clinical trials including women with PCOS $(\mathrm{n}=34)$ [59] and women with hyperandrogenism $(\mathrm{n}=8)$ [58]. Both trials examined the effects on androgens for the aqueous extract TJ-68 (equal parts Glycyrrhiza uralensis and Paeonia lactiflora), 75 grams per day for 24 weeks and 510 grams per day for 2-8 weeks respectively. In the trial including women with PCOS, mean serum testosterone was significantly reduced from $137.1 \mathrm{ng} / \mathrm{dL}( \pm 27.6)$ to $85.3 \mathrm{ng} / \mathrm{dL}( \pm 38), \mathrm{p}<0.001$ at four weeks of treatment [59]. Similar effects were observed in the women with oligomenorrhoea and hyperandrogenism which showed serum testosterone reduced from $50-160 \mathrm{ng} / \mathrm{dL}$ prior to treatment to less than $50 \mathrm{ng} / \mathrm{dL}$ [58]. However statistical significance was not reached due to the small sample size despite positive outcomes in seven out of eight participants (Table 1).

\section{Paeonia lactiflora and cinnamomum cassia}

Paeonia lactiflora combined with Cinnamomum cassia in a preparation called Unkei-to was investigated in an invitro study for ovarian production of 17-beta-oestradiol and progesterone, [42] (Table 1). Granulosa cells obtained from women undergoing IVF were examined for steroid hormone concentration following incubation with different doses over 48 hours. Oestradiol was significantly increased $(\mathrm{p}<0.01)$ following exposure to doses of $0.3 \mathrm{ug} / \mathrm{ml}$ of Unkei-to. Supporting clinical evidence was found in one clinical trial of 157 infertile women aged 17-29 years, including a subgroup of 42 women with hyperfunctioning (PCOS) oligo/amenorrhoea. Treatment with Unkei-to, 7.5 grams per day for eight weeks, demonstrated significant reductions of mean LH in the PCOS sub-group of $49.7 \%$ ( \pm 15.3$)$. Ovulation was confirmed in 30 out of 42 
oligo/amenorrheic women [57] (Table 1). Limitations however include findings based on sub-group comparisons without description of subgroup baseline characteristics (other than oligomenorrhoea). Although the same aqueous extract intervention was investigated in preclinical and clinical studies, it contained additional herbal extracts and it was irrational to attribute hormonal effects to Paeonia lactiflora and Cinnamomum cassia.

\section{Cinnamomum cassia}

An animal study compared the effectiveness of Cinnamomum cassia and the pharmaceutical Metformin on hormone concentration in rats with PCOS [48] (Table 1). Both interventions demonstrated significant improvements compared to controls at 15 days for measures of testosterone ng/ml (control 0.747 \pm 0.039 ; metformin 0.647 \pm 0.027 ; Cinnamomum cassia $0.625 \pm 0.029$ ); LH ng/ml (control $7.641 \pm$ 0.267 ; metformin $6.873 \pm 0.214$; Cinnamomum cassia $6.891 \pm 0.221$ ) and insulin resistance (HOMA-IR) (control $10.018 \pm 0.217$; metformin $7.067 \pm 0.184$ Cinnamomum cassia $8.772 \pm 0.196)(\mathrm{p}<0.05)$ [48]. The metabolic effects for Cinnamomum cassia were further demonstrated in overweight women with oligo/amenorrhoea and PCOS in a placebo controlled RCT [66] (Table 2). However, although the RCT had low risks for bias, it was a pilot study primarily investigating feasibility. Outcomes were promising for metabolic profile in PCOS however the sample size was small and the authors recommended further studies.

\section{Summary of results}

This review includes 18 preclinical laboratory based studies and 15 clinical trials. We found reproductive endocrine effects in oligo/amenorrhoea, hyperandrogenism and/or PCOS for six herbal medicines. The quality of evidence, as determined by the volume of pre-clinical studies and the methodological quality of clinical trials, was highest for the herbal medicines Vitex agnus-castus, Cimicifuga racemosa and Cinnamomum cassia, for which there were laboratory and/or animal studies demonstrating endocrine mechanisms of action consistent with clinical outcomes shown in RCT's with low risks for bias. However, replicated RCT data was only found for one herbal medicine, Cimicifuga racemosa.

Evidence for Tribulus terrestris, Glycyrrhiza spp. alone and in combination with Paeonia lactiflora and Paeonia lactiflora with Cinnamomum cassia was limited by the volume of laboratory and animal studies, with only one to two studies found for each herb or herbal combination. There was supporting clinical data, however many were small single arm, open label studies measuring endocrine effects in healthy women. Evidence for these herbal medicines is preliminary and in an emergent phase.

\section{Discussion}

This review synthesises the evidence for mechanisms of effect for herbal medicine in oligo/amenorrhoea, hyperandrogenism and PCOS. Laboratory, animal and clinical studies demonstrate that the herbal medicines Vitex agnus-castus, Cimicifuga racemosa and Tribulus terrestris initiate endocrine effects in the pituitary as measured by lowered prolactin and LH and raised FSH. Four herbal medicines, Tribulus terrestris, Glycyrrhiza spp., (alone and in combination with Paeonia lactiflora), Paeonia lactiflora (in combination with Cinnamomum cassia) and Cinnamomum cassia demonstrated morphological changes in polycystic ovaries and steroidogenesis, including reduced ovarian volume and cysts, lowered androgens, improved insulin sensitivity and increased oestradiol.

Clinical investigations found no adverse effects for the six herbal medicines included in this review (Table 2). A comparative study investigating the pharmaceutical Bromocriptine and the herbal medicine Vitex agnuscastus found no side effects associated Vitex agnus-castus compared to $12.5 \%$ of participants taking Bromocriptine reporting nausea and vomiting [63]. No studies comparing the effectiveness for herbal medicines and the oral contraceptive pill in PCOS, oligo/amenorrhoea and hyperandrogenism were found.

Herbal medicine may present a treatment option for women with oligo/amenorrhoea, hyperandrogenism and PCOS as an adjunct or alternative treatment to pharmaceuticals with a high degree of acceptability by women with PCOS [6]. Preliminary evidence for equivalent treatment effects were found for the two pharmaceuticals and three herbal medicines. These were bromocriptine, in the management of hyperprolactinaemia andVitex agnuscastus and clomiphene for infertility and ovulation induction and Cimicifuga racemosa and Tribulus terrestris. Herbal medicine had positive adjunct effects with the pharmaceuticals Spirinolactone in the management of hyperandrogenism (Glycyrrhiza Spp.), and clomiphene for PCOS related infertility (Cimicifuga racemosa). It is important however to highlight that evidence was provided by a limited number of clinical studies, some with significant risks for bias; particularly Tribulus terrestris, Glycyrrhiza glabra alone and in combination with Paeonia lactiflora and Paeonia lactiflora in combination with Cinnamomum cassia.

Selection of herbal medicines for the management of PCOS often includes the combined prescription of Glycyrrhiza spp. and Paeonia lactiflora [72-75]. We found preliminary evidence for this combination for hyperandrogenism only, and the evidence was more robust for Glycyrrhiza spp. alone than when combined with Paeonia lactiflora. Comparatively, our findings for the combination of Peaonia lactiflora and Cinnamomum cassia demonstrated no change in androgen concentration, suggesting that the anti- 
androgen activity in the Glycyrrhiza spp. and Paeonia lactiflora combination more likely attributable to Glycyrrhiza spp. However our findings may be complicated by the aqueous extraction methods used in the Paeonia lactiflora and Cinnamomum cassia combination and the preclinical studies into the Glycorrhizza spp and Paeonia lactiflora combination. More research into the antiandrogen effects of the combination Glycyrrhiza spp. and Paeonia lactiflora is needed to clarify the antiandrogen mechanism particularly if this herbal combination remains cornerstone herbal management for hyperandrogenism.

This review has some limitations. We used a methodological approach which was deductive and not consistent with traditional rationale for herbal selection. Our inclusion criteria for clinical studies were specific and relied upon our identification of herbal medicines with preclinical (laboratory based) evidence explaining the mechanisms of reproductive endocrinological effects in oligo/amenorrhoea, hyperandrogenism and PCOS. Clinical studies were excluded from this review due to the absence of evidence for whole herbal extracts. This was the case for Camellia sinensis (green tea) for which only one laboratory study investigated the effects of injecting epigallocatechin, a catechin found in green tea in animals [76]. High quality clinical evidence for Camellia sinensis was not presented in this review due to the absence of pre-clinical data explaining the mechanism for effect for the whole herbal extract [77]. Mentha spicata (spearmint) was another herbal medicine excluded from this review despite the availability of high quality clinical evidence demonstrating testosterone lowering effects in women with PCOS [78]. We found no laboratory evidence describing the mechanism of action for Mentha spicata in hyperandrogenism. Camilla sinensis and Mentha spicata are examples of herbal medicines excluded from this review due to not meeting the inclusion criteria. Studies investigating western herbal medicines excluded from this review are provided in Tables 3, 4 and 5.

Our search strategy may have restricted access due to limited search terms. We didn't include alternative spelling of oestrogen and additional search terms for herbal medicine could have been included to increase sensitivity of the search.

This study synthesises the evidence for reproductive endocrine effects for six whole herbal medicine extracts that may be used to treat PCOS and associated oligo/ amenorrhoea and hyperandrogenism. The findings were intended to add to clinicians understanding for the mechanisms of action for herbal medicine for treatment in these common conditions and reveal herbal medicines with reproductive endocrinological effects, currently demonstrated in scientific literature.

\section{Conclusions}

Preclinical and clinical studies provide preliminary evidence that six herbal medicines may have beneficial effects for women with oligo/amenorrhea, hyperandrogenism and PCOS. The quality of the evidence is variable and strongest for Vitex agnus-castus and Cimicifuga racemosa in the management of oligo/amenorrhea and infertility associated with PCOS; and Cinnamomum cassia for improving metabolic hormones in PCOS. Evidence for Tribulus terrestris, Glycyrrhiza spp. alone and in combination with Paeonia lactiflora and Paeonia lactiflora combined with Cinnamon cassia is promising but in an emergent phase. Further investigations into the mechanisms of effect for herbal extracts are needed to complete our understanding of the reproductive endocrinological effects for herbal medicine for these common conditions.

\section{Competing interests}

The authors declare that they have no competing interests.

\section{Authors' contributions}

$S A, J A, C S$ and $A B$ conceived of the study and participated in its design and coordination. SA carried out the search of the literature. SA, JA and CS participated in study inclusion or exclusion. SA performed data extraction and $C S, J A$ and $A B$ reviewed the quality of data. $S A, J A$ and $A B$ designed and edited the tables. All authors read and approved the final manuscript.

\section{Authors' information}

$S A$ is a doctoral research student and CAS, JAB and $A B$ are supervisory personnel. The submission processing fee was provided by the University of Western Sydney as part of an academic institutional membership.

\section{Acknowledgements}

The authors would like to acknowledge the contribution of the late Dr Tini Gruner of Southern Cross University for her review of SA's translation of the three German clinical studies.

\section{Author details}

${ }^{1}$ National Institute of Complementary Medicine, University of Western, Locked Bag 1797, Penrith South, NSW 2751 Sydney, Australia. ${ }^{2}$ School of Women's and Children's Health, University of New South Wales, Sydney, Sydney, Australia. ${ }^{3}$ National Institute of Complementary Medicine (NICM), University of Western Sydney, Sydney, Australia.

Received: 17 July 2014 Accepted: 9 December 2014 Published: 18 December 2014

\section{References}

1. March WA, Moore VM, Willson KJ, Phillips DI, Norman RJ, Davies MJ: The prevalence of polycystic ovary syndrome in a community sample assessed under contrasting diagnostic criteria. Hum Reprod 2010, 25(2):544-551.

2. Teede HJ, Misso ML, Deeks AA, Moran $\sqcup$, Stuckey BG, Wong JL, Norman RJ, Costello MF, Guideline Development Groups: Assessment and management of polycystic ovary syndrome: summary of an evidence-based guideline. Med J Aust 2011, 195(6):65.

3. ESHRE: Consensus on women's health aspects of polycystic ovary syndrome (PCOS). Hum Reprod 2012, 27(1):14-24.

4. Messinis IE: Ovulation induction: a mini review. Hum Reprod 2005, 20(10):2688-2697.

5. Tang T, Lord JM, Norman RJ, Yasmin E, Balen AH: Insulin-sensitising drugs (metformin, rosiglitazone, pioglitazone, D-chiro-inositol) for women with polycystic ovary syndrome, oligo amenorrhoea and subfertility. Cochrane Database Syst Rev 2010, 1:2-12. 
6. Sills ES, Perloe M, Tucker MJ, Kaplan CR, Genton MG, Schattman GL: Diagnostic and treatment characteristics of polycystic ovary syndrome: descriptive measurements of patient perception and awareness from 657 confidential self-reports. BMC Womens Health 2001, 1(1):3.

7. Holden S, Davis R, Yeh G: Pregnant Women's Use of Complementary \& Alternative Medicine in the United States. J Alternative Compl Med 2014, 20(5):A120.

8. Lunny CA, Fraser SN: The Use of Complementary and Alternative Medicines Among a Sample of Canadian Menopausal-Aged Women. J Midwifery Womens Health 2010, 55(4):335-343.

9. Bishop JL, Northstone K, Green JR, Thompson EA: The use of complementary and alternative medicine in pregnancy: data from the Avon Longitudinal Study of Parents and Children (ALSPAC). Complement Ther Med 2011, 19(6):303-310.

10. Nordeng H, Bayne K, Havnen GC, Paulsen BS: Use of herbal drugs during pregnancy among 600 Norwegian women in relation to concurrent use of conventional drugs and pregnancy outcome. Complement Ther Clin Pract 2011, 17(3):147-151.

11. Smith CA, Bateson DJ, Weisberg E: A survey describing the use of complementary therapies and medicines by women attending a family planning clinic. BMC Complement Altern Med 2013, 13(1):224.

12. Stankiewicz M, Smith C, Alvino H, Norman R: The use of complementary medicine and therapies by patients attending a reproductive medicine unit in South Australia: a prospective survey. Aust New Zeal J Obstet Gynaecol 2007, 47(2):145-149.

13. Ren $M Q$, Kuhn G, Wegner J, Chen J: Isoflavones, substances with multibiological and clinical properties. Eur J Nutr 2001, 40(4):135-146.

14. Whitten PL, Naftolin F: Reproductive actions of phytoestrogens. Baillieres Clin Endocrinol Metab 1998, 12(4):667-690.

15. Wolff MS, Teitelbaum SL, Pinney SM, Windham G, Liao L, Biro F, Kushi LH, Erdmann C, Hiatt RA, Rybak ME, Calafat AM: Investigation of relationships between urinary biomarkers of phytoestrogens, phthalates, and phenols and pubertal stages in girls. Environ Health Perspect 2010, 118(7):1039-1046.

16. Wei $W$, Zhao $H$, Wang A, Sui $M$, Liang $K$, Deng $H, M a ~ Y$, Zhang $Y$, Zhang $H$, Guan Y: A clinical study on the short-term effect of berberine in comparison to metformin on the metabolic characteristics of women with polycystic ovary syndrome. Eur J Endocrinol 2012, 166(1):99-105.

17. Francis G, Kerem Z, Makkar HPS, Becker K: The biological action of saponins in animal systems: a review. Br J Nutr 2002, 88(6):587-605.

18. Grant P, Ramasamy S: An Update on Plant Derived Anti-Androgens. Int J Endocrinol Metabol 2012, 2012(2, Spring):497-502.

19. Norman RJ, Dewailly D, Legro RS, Hickey TE: Polycystic ovary syndrome. Lancet 2007, 370(9588):685-697.

20. ESHRE: Consensus on infertility treatment related to polycystic ovary syndrome. Hum Reprod 2008, 23(3):462-477.

21. Brown J, Farquhar C, Beck J, Boothroyd C, Hughes E: Clomiphene and antioestrogens for ovulation induction in PCOS. Cochrane Database Syst Rev 2009. doi:10.1002/14651858.CD002249.pub4.

22. Polson D, Kiddy DS, Mason HD, Franks S: Induction of ovulation with clomiphene citrate in women with polycystic ovary syndrome: the difference between responders and nonresponders. Fertil Steril 1989, 51(1):30-34.

23. Kousta $E$, White $D$, Franks $S$ : Modern use of clomiphene citrate in induction of ovulation. Hum Reprod Update 1997, 3(4):359-365.

24. Tang T, Glanville J, Hayden CJ, White D, Barth JH, Balen AH: Combined lifestyle modification and metformin in obese patients with polycystic ovary syndrome. A randomized, placebo-controlled, double-blind multicentre study. Hum Reprod 2006, 21(1):80-89.

25. Williamson E: Synergy and other interactions in phytomedicines. Phytomedicine 2001, 8(5):401-409.

26. Mills S, Bone K: Principles and Practice of Phytotherapy. London, England: Churchill Livingstone Harcourt Publishers; 2000.

27. Wardle JL, Adams J, Lui C-W: A qualitative study of naturopathy in rural practice: A focus upon naturopaths' experiences and perceptions of rural patients and demands for their services. BMC Health Serv Res 2010, 10(1):185

28. Steel A, Wardle J, Diezel H, Johnstone K, Adams J: Educating for collaboration: The outcomes of an interprofessional education workshop for complementary and alternative maternity care providers. Adv Integr Med 2014, 1(1):17-24.
29. Teede H, Gibson-Helm M, Norman RJ, Boyle J: Polycystic Ovary Syndrome: Perceptions and Attitudes of Women and Primary Health Care Physicians on Features of PCOS and Renaming the Syndrome. J Clin Endocrinol Metabol 2013, 99(1):E107-E111.

30. ESHRE: Revised 2003 consensus on diagnostic criteria and long-term health risks associated with polycystic ovary syndrome. Fertil Steril 2004, 81(1):19-25.

31. Legro RS, Zaino RJ, Demers LM, Kunselman AR, Gnatuk CL, Williams NI, Dodson WC: The effects of metformin and rosiglitazone, alone and in combination, on the ovary and endometrium in polycystic ovary syndrome. Am J Obstet Gynecol 2007, 196(4):402.e1-402.e11.

32. Conway $\mathrm{G}$, Honour J, Jacobs $\mathrm{H}$ : Heterogeneity of the polycystic ovary syndrome: clinical, endocrine and ultrasound features in 556 patients. Clin Endocrinol (Oxf) 1989, 30(4):459-470.

33. Balen $\mathrm{AH}$, Tan $\mathrm{SL}$, Jacobs HS: Hypersecretion of luteinising hormone: a significant cause of infertility and miscarriage. BJOG 1993, 100(12):1082-1089.

34. Legro RS, Kunselman AR, Dodson WC, Dunaif A: Prevalence and predictors of risk for type 2 diabetes mellitus and impaired glucose tolerance in polycystic ovary syndrome: a prospective, controlled study in 254 affected women. J Clin Endocrinol Metabol 1999, 84(1):165-169.

35. Luciano A, Chapler F, Sherman B: Hyperprolactinemia in polycystic ovary syndrome. Fertil Steril 1984, 41(5):719-725

36. Jarry H, Spengler B, Porzel A, Schmidt J, Wuttke W, Christoffel V: Evidence for estrogen receptor beta-selective activity of Vitex agnus-castus and isolated flavones. Planta Med 2003, 69(10):945-946.

37. Jarry H, Harnischfeger G, Düker E: Studies on the endocrine effects of the contents of Cimicifuga racemosa. In vitro binding of compounds to estrogen receptors. Planta Med 1985, 51(4):316.

38. Jarry H, Spengler B, Wuttke W, Christoffel V: In vitro assays for bioactivityguided isolation of endocrine active compounds in Vitex agnus-castus. Maturitas 2006, 55:S26-S36.

39. Jarry H, Leonhardt S, Gorkow C, Wuttke W: In vitro prolactin but not LH and FSH release is inhibited by compounds in extracts of Agnus castus: direct evidence for a dopaminergic principle by the dopamine receptor assay. Exp Clin Endocrinol Diabetes 2009, 102(06):448-454.

40. Meier B, Berger D, Hoberg E, Sticher O, Schaffner W: Pharmacological activities of Vitex agnus-castus extracts in vitro. Phytomedicine 2000, 7(5):373-381.

41. Sliutz G, Speiser P, Schultz AM, Spona J, Zeillinger R: Agnus-castus extracts inhibit prolactin secretion of rat pituitary cells. Horm Metab Res 1993, 25(5):253-255

42. Sun WS, Imai A, Tagami K, Sugiyama M, Furui T, Tamaya T: In vitro stimulation of granulosa cells by a combination of different active ingredients of unkei-to. Am J Chin Med 2004, 32(4):569-578.

43. Wuttke W, Jarry H, Christoffel V, Spengler B, Seidlová-Wuttke D: Chaste tree (Vitex agnus-castus). Pharmacology and clinical indications. Phytomedicine 2003, 10(4):348-357.

44. Zierau O, Bodinet C, Kolba S, Wulf M, Vollmer G: Antiestrogenic activities of Cimicifuga racemosa extracts. J Steroid Biochem Mol Biol 2002, 80(1):125-130.

45. Düker EM, Kopanski L, Jarry H, Wuttke W: Effects of extracts from Cimicifuga racemosa on gonadotropin release in menopausal women and ovariectomized rats. Planta Med 1991, 57(5):420-424.

46. Dehghan A, Esfandiari A, Bigdeli SM: Alternative Treatment of Ovarian Cysts with Tribulus terrestris Extract: A Rat Model. Reprod Domest Anim 2012, 47(1):e12-e15.

47. Esfandiari A, Dehghan A, Sharifi S, Najafi B, Vesali E: Effect of Tribulus terrestris extract on ovarian activity in immature Wistar rat: a histological evaluation. J Anim Vet Adv 2011, 10(7):883-886.

48. Heibashy M, Mazen G, Shahin M: Metabolic Changes and Hormonal Disturbances in Polycystic Ovarian Syndrome Rats and the Amelioration Effects of Metformin and/or Cinnamon Extraction. J Am Sci 2013, 9(12):p54-p62.

49. Ibrahim N, Shalaby AS, Farag RS, Elbaroty GS, Nofal SM, Hassan EM: Gynecological efficacy and chemical investigation of Vitex agnus-castus L. fruits growing in Egypt. Nat Prod Res 2008, 22(6):537-546.

50. Lee JC, Pak SC, Lee SH, Lim SC, Bai YH, Jin CS, Kim JS, Na CS, Bae CS, Oh KS: The effect of herbal medicine on nerve growth factor in estradiol valerate-induced polycystic ovaries in rats. Am J Chin Med 2003, 31(06):885-895.

51. Martino-Andrade AJ, Morais RN, Spercoski KM, Rossi SC, Vechi MF, Golin M, Lombardi NF, Greca CS, Dalsenter PR: Effects of Tribulus terrestris on endocrine sensitive organs in male and female Wistar rats. J Ethnopharmacol 2010, 127(1):165-170. 
52. Seidlova-Wuttke D, Hesse O, Jarry H, Christoffel V, Spengler B, Becker T, Wuttke W: Evidence for selective estrogen receptor modulator activity in a black cohosh (Cimicifuga racemosa) extract: comparison with estradiol-17beta. Eur J Endocrinol 2003, 149(4):351-362.

53. Takeuchi T, Nishii O, Okamura T, Yaginuma T: Effect of traditional herbal medicine, shakuyaku-kanzo-to on total and free serum testosterone levels. Am J Chin Med 1989, 17(1-2):35-44.

54. Armanini D, Castello R, Scaroni C, Bonanni G, Faccini G, Pellati D, Bertoldo A, Fiore C, Moghetti P: Treatment of polycystic ovary syndrome with spironolactone plus licorice. Eur J Obstet Gynecol Reprod Biol 2007, 131(1):61-67.

55. Armanini D, Mattarello MJ, Fiore C, Bonanni G, Scaroni C, Sartorato P, Palermo M: Licorice reduces serum testosterone in healthy women. Steroids 2004, 69(11-12):763-766.

56. Milanov S, Maleeva A, Tashkov M: Tribestan effect on the concentration of some hormones in the serum of healthy subjects. Sofia, Bulgaria: Company documentation, Chemical Pharmaceutical Research Institute; 1981.

57. Ushiroyama T, Ikeda A, Sakai M, Hosotani T, Suzuki Y, Tsubokura S, Ueki M: Effects of unkei-to, an herbal medicine, on endocrine function and ovulation in women with high basal levels of luteinizing hormone secretion. J Reprod Med 2001, 46(5):451-456.

58. Yaginuma Tl, Yasui R, Arai H, Kawabata T: Effect of traditional herbal medicine on serum testosterone levels and its induction of regular ovulation in hyperandrogenic and oligomenorrheic women. Nippon Sanka Fujinka Gakkai Zasshi 1982, 34(7):939.

59. Takahashi K, Kitao M: Effect of TJ-68 (shakuyaku-kanzo-to) on polycystic ovarian disease. Int J Fertil Menopausal Stud 1994, 39(2):69.

60. Tabakova P, Dimitrov M, Tashkov B: Clinical studies on the preparation Tribestan in women with endocrine infertility or menopausal syndrome. Sofia, Bulgaria: 1st Obstetrical and Gynecological Hospital; 1984.

61. Gerhard I, Patek A, Monga B, Blank A, Gorkow C: Mastodynon ${ }^{\circledR}$ for Female Infertility. Randomized placebo controlled, clinical double-blind study. Forschende Komplementärmedizin/Res Compl Med 1998, 5(6):272-278.

62. Bergmann J, Luft B, Boehmann S, Runnebaum B, Gerhard I: The efficacy of the complex medication Phyto-Hypophyson $L$ in female, hormonerelated sterility. A randomized, placebo-controlled clinical double-blind study. Forschende Komplementärmedizin und klassische Naturheilkunde. Res Compl Nat Classical Med 2000, 7(4):190

63. Kilicdag E, Tarim E, Bagis T, Erkanli S, Aslan E, Ozsahin K, Kuscu E: Fructus agni casti and bromocriptine for treatment of hyperprolactinemia and mastalgia. Int J Gynecol Obstet 2004, 85(3):292-293.

64. Milewicz A, Gejdel E, Sworen H, Sienkiewicz K, Jedrzejak J, Teucher T, Schmitz $\mathrm{H}$ : Vitex agnus castus extract in the treatment of luteal phase defects due to latent hyperprolactinemia. Results of a randomized placebo-controlled double-blind study. Arzneimittel-Forschung (Drug Res) 1993, 64(7):752-756.

65. Shahin AY, Ismail AM, Zahran KM, Makhlouf AM: Adding phytoestrogens to clomiphene induction in unexplained infertility patients - a randomized trial. Reprod Biomed Online 2008, 16(4):580-588.

66. Wang JG, Anderson RA, Graham GM III, Chu MC, Sauer MV, Guarnaccia MM, Lobo RA: The effect of cinnamon extract on insulin resistance parameters in polycystic ovary syndrome: a pilot study. Fertil Steril 2007, 88(1):240-243.

67. Kamel HH: Role of phyto-oestrogens in ovulation induction in women with polycystic ovarian syndrome. Eur J Obstet Gynecol Reprod Biol 2013, 168(1):60-63.

68. Shahin AY, Mohammed SA: Adding the phytoestrogen Cimicifugae Racemosae to clomiphene induction cycles with timed intercourse in polycystic ovary syndrome improves cycle outcomes and pregnancy rates-a randomized trial. Gynecol Endocrinol 2014, 30(7):505-510.

69. Jarry H, Metten M, Spengler B, Christoffel V, Wuttke W: In vitro effects of the Cimicifuga racemosa extract BNO 1055. Maturitas 2003, 44(Supplement 1):S31-S38.

70. Webster D, Lu J, Chen SN, Farnsworth NR, Wang ZJ: Activation of the $\mu$-opiate receptor by Vitex agnus-castus methanol extracts: Implication for its use in PMS. J Ethnopharmacol 2006, 106(2):216-221.

71. Chan CC, Koo MW, Ng EH, Tang OS, Yeung WS, Ho PC: Effects of Chinese green tea on weight, and hormonal and biochemical profiles in obese patients with polycystic ovary syndrome - a randomized placebo-controlled trial. J Soc Gynecol Investig 2006, 13(1):63-68.
72. Kao Y-H, Hiipakka RA, Liao S: Modulation of endocrine systems and food intake by green tea epigallocatechin gallate 1. Endocrinology 2000, 141(3):980-987.

73. Akdoğan M, Tamer MN, Cüre E, Cüre MC, Köroğlu BK, Delibaş N: Effect of spearmint (Mentha spicata Labiatae) teas on androgen levels in women with hirsutism. Phytother Res 2007, 21(5):444-447.

74. Grant P: Spearmint herbal tea has significant anti-androgen effects in polycystic ovarian syndrome. A randomized controlled trial. Phytother Res 2010, 24(2):186-188.

75. Kudolo GB, Wang W, Javors M, Blodgett J: The effect of the ingestion of Ginkgo biloba extract (EGb 761) on the pharmacokinetics of metformin in non-diabetic and type 2 diabetic subjects-A double blind placebocontrolled, crossover study. Clin Nutr 2006, 25(4):606-616.

76. Chen J-T, Tominaga K, Sato Y, Anzai H, Matsuoka R: Maitake mushroom (Grifola frondosa) extract induces ovulation in patients with polycystic ovary syndrome: a possible monotherapy and a combination therapy after failure with first-line clomiphene citrate. J Alternative Compl Med 2010, 16(12):1295-1299.

77. Phipps WR, Martini MC, Lampe JW, Slavin JL, Kurzer MS: Effect of flax seed ingestion on the menstrual cycle. (J Clin Endocrinol Metabo 1993, 77(5):1215-1219.

78. Lampe J, Martini MC, Kurzer MS, Adlercreutz H, Slavin JL: Urinary lignan and isoflavonoid excretion in premenopausal women consuming flaxseed powder. Am J Clin Nutr 1994, 60(1):122-128.

79. Hutchins AM, Martini MC, Olson BA, Thomas W, Slavin JL: Flaxseed consumption influences endogenous hormone concentrations in postmenopausal women. Nutr Cancer 2001, 39(1):58-65.

80. Frische EJ, Hutchins AM, Martini MC, Thomas W, Slavin JL: Effect of flaxseed and wheat bran on serum hormones and lignan excretion in premenopausal women. J Am Coll Nutr 2003, 22(6):550-554.

81. Melo E, Bertero EB, Rios LA, Mattos D Jr: Evaluating the efficiency of a combination of Pygeum africanum and stinging nettle (Urtica dioica) extracts in treating benign prostatic hyperplasia (BPH): double-blind, randomized, placebo controlled trial. Int Braz J Urol 2002, 28(5):418-425.

82. Yang J, Te AE: Saw palmetto and finasteride in the treatment of category-III prostatitis/chronic pelvic pain syndrome. Curr Urol Rep 2005, 6(4):290-295.

83. Morgia G, Mucciardi G, Madonia M, Castelli T, Favilla V, Magno C: Treatment of chronic prostatitis/chronic pelvic pain syndrome (CP/CPPS) with Serenoa repens plus selenio and licopene (Profluss ${ }^{\circledR}$ ): a randomized multicenter placebo-controlled study. J Urol 2008, 179(4):32.

84. Casner PR, Bent S, Kane C, Shinohara K: Saw palmetto for benign prostatic hyperplasia. N Engl J Med 2006, 354(18):1950-1951.

85. Huseini HF, Larijani B, Heshmat R, Fakhrzadeh H, Radjabipour B, Toliat T, Raza M: The efficacy of Silybum marianum (L.) Gaertn. (silymarin) in the treatment of type II diabetes: a randomized, double-blind, placebo-controlled, clinical trial. Phytother Res 2006, 20(12):1036-1039.

86. Jalilian N, Modarresi M, Rezaie M, Ghaderi L, Bozorgmanesh M: Phytotherapeutic Management of Polycystic Ovary Syndrome: Role of Aerial Parts of Wood Betony (Stachys lavandulifolia). Phytother Res 2013, 27(11):1708-1713

87. Najafipour F, Rahimi AO, Mobaseri M, Agamohamadzadeh N, Nikoo A, Aliasgharzadeh A: Therapeutic effects of stinging nettle (Urtica dioica) in women with Hyperandrogenism. Int J Current Res Acad Rev 2014, 2(7):153-160.

88. Hryb D, Khan MS, Romas NA, Rosner W: The effect of extracts of the roots of the stinging nettle. Planta Med 1995, 61(01):31-32.

89. Schöttner M, Ganßer D, Spiteller G: Lignans from the Roots of Urtica spp. Planta Med 1997, 63(06):529-532.

90. Zhang Q, Li L, Liu L, Li Y, Yuan L, Song L, Wu Z: Effects of the polysaccharide fraction of Urtica fissa on castrated rat prostate hyperplasia induced by testosterone propionate. Phytomedicine 2008, 15(9):722-727.

91. Hajhashemi $V$, Klooshani $V$ : Antinociceptive and anti-inflammatory effects of Urtica dioica leaf extract in animal models. Avicenna J Phytomedicine 2013, 3(2):193-200.

92. Loch $E$, Böhnert K, Peeters M: The treatment of menstrual disorders with Vitex agnus-castus tincture. Der Frauenarzt 1991, 32(8):867-870.

93. Loch E, Selle H, Boblitz N: Treatment of premenstrual syndrome with a phytopharmaceutical formulation containing Vitex agnus castus. J Womens Health Gend Based Med 2000, 9(3):315-320. 
94. Ma L, Lin S, Chen R, Zhang Y, Chen F, Wang X: Evaluating therapeutic effect in symptoms of moderate-to-severe premenstrual syndrome with Vitex agnus castus (BNO 1095) in Chinese women. Aust New Zeal J Obstet Gynaecol 2010, 50(2):189-193.

95. Schellenberg R: Treatment for the premenstrual syndrome with agnus castus fruit extract: prospective, randomised, placebo controlled study. BMJ (Clin Res ed) 2001, 134-137.

96. Lauritzen $\mathrm{CH}$, Reuter HD, Repges R, Böhnert KJ, Schmidt U: Treatment of premenstrual tension syndrome with Vitex agnus castus controlled, double-blind study versus pyridoxine. Phytomedicine 1997, 4(3):183-189.

97. He Z, Chen R, Zhou Y, Geng L, Zhang Z, Chen S, Yao Y, Lu J, Lin S: Treatment for premenstrual syndrome with Vitex agnus castus: A prospective, randomized, multi-center placebo controlled study in China. Maturitas 2009, 63(1):99-103.

98. Wuttke W, Splitt G, Gorkow C, Sieder C: Treatment of cyclical mastalgia: Results of a randomised, placebo- controlled, double-blind study: Objective. Obstet Gynecol 1997, 57(10):569-574.

99. Halaska M, Beles P, Gorkow C, Sieder C: Treatment of cyclical mastalgia with a solution containing a Vitex agnus castus extract: results of a placebo-controlled double-blind study. Breast 1999, 8(4):175-181.

100. Wuttke W, Seidlova-Wuttke D, Gorkow C: The Cimicifuga preparation BNO 1055 vs. conjugated estrogens in a double-blind placebo-controlled study: effects on menopause symptoms and bone markers. Maturitas 2003, 44:S67-S77.

101. Yamada K, Kanba S, Yagi G, Asai M: Herbal medicine (Shakuyaku-kanzo-to) in the treatment of risperidone-induced amenorrhea. J Clin Psychopharmacol 1999, 19(4):380.

102. Ohtsu H, Xiao Z, Ishida J, Nagai M, Wang HK, Itokawa H, Su CY, Shih C, Chiang T, Chang E: Antitumor agents. 217. Curcumin analogues as novel androgen receptor antagonists with potential as anti-prostate cancer agents. J Med Chem 2002, 45(23):5037-5042.

103. Zangeneh FZ, Minaee B, Amirzargar A, Ahangarpour A, Mousavizadeh K: Effects of chamomile extract on biochemical and clinical parameters in a rat model of polycystic ovary syndrome. J Reprod Infertil 2010, 11(3):169.

104. Akdogan M, Ozguner M, Kocak A, Oncu M, Cicek E: Effects of peppermint teas on plasma testosterone, follicle-stimulating hormone, and luteinizing hormone levels and testicular tissue in rats. Urology 2004 64(2):394-398

105. Gebhardt R: Antioxidative, antiproliferative and biochemical effects in HepG2 cells of a homeopathic remedy and its constituent plant tinctures tested separately or in combination. Arzneimittel Forschung 2003, 53(12):823-830.

106. Burdette JE, Liu J, Chen S, Fabricant DS, Piersen CE, Barker EL, Pezzuto JM, Mesecar A, van Breemen RB, Farnsworth NR: Black cohosh acts as a mixed competitive ligand and partial agonist of the serotonin receptor. J Agric Food Chem 2003, 51(19):5661-5670.

107. Whitehead SA, Lacey M: Phytoestrogens inhibit aromatase but not $17 \beta$ hydroxysteroid dehydrogenase (HSD) type 1 in human granulosa luteal cells: evidence for FSH induction of $17 \beta$ HSD. Hum Reprod 2003, 18(3):487-494.

108. Zhao L, Li W, Han F, Hou L, Baillargeon J-P, Kuang H, Wang Y, Wu X: Berberine reduces insulin resistance induced by dexamethasone in theca cells in vitro. Fertil Steril 2011, 95(1):461-463.

109. Kostova I, Dinchev D: Saponins in Tribulus terrestris - Chemistry and bioactivity. Phytochem Rev 2005, 4(2-3):111-137.

110. Takeuchi T, Nishii O, Okamura T, Yaginuma T: Effect of paeoniflorin, glycyrrhizin and glycyrrhetic acid on ovarian androgen production. Am J Chin Med 1991, 19(1):73-78.

doi:10.1186/1472-6882-14-511

Cite this article as: Arentz et al: Herbal medicine for the management of polycystic ovary syndrome (PCOS) and associated oligo/amenorrhoea and hyperandrogenism; a review of the laboratory evidence for effects with corroborative clinical findings. BMC Complementary and Alternative Medicine 2014 14:511.

\section{Submit your next manuscript to BioMed Central and take full advantage of:}

- Convenient online submission

- Thorough peer review

- No space constraints or color figure charges

- Immediate publication on acceptance

- Inclusion in PubMed, CAS, Scopus and Google Scholar

- Research which is freely available for redistribution

Submit your manuscript at www.biomedcentral.com/submit 